\title{
Ionic Radii from Experimental Activities and Simple Statistical-Mechanical Theories for Strong Electrolytes with Small
} Bjerrum Parameters

\author{
TORBEN SMITH SØRENSEN, PETER SLOTH and MICHAEL SCHRØDER
}

Fysisk-Kemisk Institut, DTH 206, DK-2800 Lyngby, Denmark

It is shown, that reasonable distances of closest approach between ions in aqueous solutions can be calculated from data for activity coefficients in dilute electrolyte solutions, if the usual DebyeHückel formula is used in connection with an "excluded volume" term (DHEV-model). The contact distances may be evaluated from the formula and fittings of Guggenheim by solving a cubic equation. Two possibilities are investigated, DHEV I with all kinds of excluded volumes and DHEV II with excluded volume between cations and anions only in accordance with the specific interaction principle of Brønsted. The difference is not great, though DHEV II distances are somewhat greater than DHEV I distances.

Ionic Lattice/Excluded Volume models are also considered using Madelung constants for a $\mathrm{NaCl}$ lattice for 1:1 electrolytes and for $\mathrm{a} \mathrm{CaF}_{2}$ lattice for 2:1 electrolytes. For ILEV I and ILEV II (without and with the Brønsted assumption) the contact distances differ widely, and only the ILEV I distances are close to the distances calculated from DHEV I or DHEV II.

The Debye-Hückel/Excluded Volume model can be derived as a limiting case in a Bogoliubovexpansion for small plasma-parameters using the BBGKY-hierarchy for the ionic distribution functions, when we further restrict ourselves to the case of a symmetric electrolyte with ions of equal size and to the case of small Bjerrum parameters (no ion pair formation). The DHEV model can also be seen as a limiting case of the mean spherical approximation (MSA) model of Lebowitz et al. There seems to be no basis for the Brønsted assumption in rigorous statisticalmechanical theory.

At higher electrolyte concentrations (0.1-1 $\mathrm{mol} / \mathrm{dm}^{3}$ ), no rigorous theory exists, although as well ILEV as the Bogoliubov expansion and the MSA model can be fitted with some success. A realistic model would have to take into account dielectric lowering and short-range forces from the water molecules. However, we present semiempirical formulae for easy calculation of activity coefficients of various $1: 1$ and $2: 1$ electrolytes at various temperatures. We also discuss the temperature variation of the semi-empirical contact distances in the light of the water theory of Bernal and Fowler.

The theory of very dilute solutions of strong electrolytes was proposed first by Milner ${ }^{1-4}$ and later in a more simple form by Debye and Hückel $^{5}$ in 1923. From the thirties till now, the derivations of Debye and Hückel have been criticised severely for lack of statistical-mechanical rigour, but it was not before 1950 , that an alternative derivation in terms of a cluster expansion was carried out by Mayer. ${ }^{6}$ In this way, the limiting law of Debye and Hückel was strictly verified.

As late as 1968 , Résibois ${ }^{7}$ wrote the following statement: "unfortunately, the considerable effort which has been put into this problem has not resulted in much interest. No rigorous theory is presently available beyound the limiting-law region". In hindsight, Resibois seems here to be too pessimistic. From 1966, Schmitz $^{8-9}$ devised elegant expansion methods in the so-called plasma parameter by an extension of Bogoliubov's perturbation method for the BBGKY-hierarchy 
(linking n'th order ionic distribution functions to distribution functions of order $n+1$ ). The results of Schmitz are neatly summarized in the monograph of Falkenhagen. ${ }^{10}$ Schmitz calculated the first deviations from the limiting law of MilnerDebye-Hückel.

Later on, the so-called integral equation methods have become more popular. The first approach of Kirkwood and Poirier ${ }^{11}$ used the BBGKY-hierarchy with third order distribution functions expressed as products of second order distributions (pair distributions). In this way, one may break off the hierarchy and obtain explicit formulae. From the late fifties, it has become fashionable to base integral equation methods on approximations based on the so-called direct correlation function which is defined through the Ornstein-Zernike equation, see Ref. 12, section 13-5. The two most important approximations are the Percus-Yevick and the hypernetted chain equation (PY and HNC). Both models are in good agreement with molecular dynamics simulations for hard sphere fluids without charges up to densities amounting to 0.6 times the density of the closest packed spheres. In comparison, BBGKY-based formulae deviate from molecular dynamics "data" already from 0.4 times the closest-packing density, see Ref. 12, Fig. 13-6. It should be noticed, that the PY-approximation leads to very simple analytical formulae for the thermodynamical properties of uncharged hard spheres.

Around the same time of the analytical expansions of Schmitz, Rasaiah and Friedman ${ }^{13-16}$ solved the PY- and HNC-equations numerically for hard-sphere ions in a dielectric continuum with the same dielectric constant as the ions (i.e. the "primitive" model of electrolyte solutions). Most of the results tabulated, are only valid for the restricted primitive model (RPM), where the ions have equal size. Both HNC- and PYcalculations yield osmotic coefficients in reasonable agreement with values calculated by Card and Valleau ${ }^{17}$ by Monte Carlo methods. (For 1:1 electrolytes up to $2 \mathrm{~mol} / \mathrm{dm}^{3}$, see Ref. 12 , Table 15-1). HNC seemed a little "better" than PY.

Another approach is the mean spherical approximation (MSA) devised by Lebowitz and Percus ${ }^{18}$ as an extension of the hard-sphere PY-equation. Waisman and Lebowitz ${ }^{19}$ were able to show, that the restricted, primitive model (RPM) has thermodynamical properties, which can be evaluated by simple, analytical formulae bearing great resemblance to the Debye-Hückel expression when the MSA is used. The electrostatic "shielding length" in the DH theory $(1 / \kappa)$ has only to be replaced by another, more realistic, shielding length, which converges towards $1 / \kappa$ in very dilute systems.

The message here is, that the Debye-Hückel theory is not at all as bad as its reputation in statistical mechanics. Actually, as we shall see in sections 1 and 2 , the original Debye-Hückel equation (not only their limiting law!) with the addition of a simple correction for excluded volume comes, out as a limiting case from more rigorous formalisms. We call this model the DHEV-model. In section 3, we recast the distances of closest approach tabulated by Guggenheim et al. ${ }^{20-21}$ for dilute $1: 1,2: 1$ and $1: 2$ electrolytes into more realistic DHEV-distances by solving a cubic equation.

Especially, we have investigated the assumption of Brønsted concerning the "specific interaction of ions"22,23, which states that there should only be excluded volume (specific interaction in contrast to non-specific action through ionic strength) between ions of opposite sign. This principle is often quoted in monographs of electrochemistry, and it was used by one of us in two earlier papers. ${ }^{24,25}$ The principle seems not to have any basis in the statistical mechanics of ionic solutions, although it seems to be almost fulfilled for experimental data for dilute electrolyte mixtures (Ref. 26, Chap. 14). We shall see, that the DHEV-radii are only slightly greater with the Brønsted principle than without. In section 4 , however, we investigate an ioniclattice/excluded volume (ILEV) model. This model is much more sensitive to the Brønsted hypothesis, and to have ILEV contact distances similar to DHEV-distances, the Brønsted principle should not be used.

In section 5 we shall present "practical" (semi-empirical) formulae for activities of a number of salts at high concentrations and for different temperatures. The formulae are similar to the "ASPEV" (Adjusted Screened Potential/ Excluded Volume) formulae from the earlier publications, ${ }^{24,25}$ but they are simpler, and they yield a smooth transition to the DH-expression and the limiting law. 


\section{BOGOLIUBOV EXPANSION}

Bogoliubov expansion of BBGKY-hierarchy after Schmitz. The fundamental dimensionless quantities in the expansion made by Schmitz ${ }^{8,9,10}$ are the following three:

Plasma parameter between ion types no. $\mathrm{i}$ and $\mathrm{j}$

$\mu_{\mathrm{ij}}=\frac{-z_{\mathrm{i}} z_{\mathrm{j}} \cdot e_{\mathrm{o}}^{2}}{4 \pi \varepsilon k T} \cdot \kappa$

Dimensionless contact distance (or dimensionless squareroot of ionic strength)

$\eta_{\mathrm{ij}}=\kappa \cdot R_{\mathrm{ij}}$

Bjerrum parameter

$\xi_{\mathrm{ij}}=\frac{-z_{\mathrm{i}} z_{\mathrm{j}} e_{\mathrm{o}}^{2}}{4 \pi \varepsilon R_{\mathrm{ij}} \cdot k T}$

The inverse Debye-Hückel screening length is given by:

$\kappa=F \cdot \sqrt{\frac{2 I}{R T \varepsilon}}$

All formulae are in SI-units. The charge number of ion type no. $\mathrm{i}$ is $z_{\mathrm{i}}$ (integer with sign). The elementary charge is $e_{0}=F / N_{\mathrm{o}}$, with $F$ being Faraday's constant and $N_{\mathrm{o}}$ Avogadro's number. The absolute permittivity of the pure solvent is $\varepsilon$, $k=R / N_{\mathrm{o}}$ is Boltzmann's constant and $R_{\mathrm{ij}}$ is the sum of the hard-sphere radii for type $i$ and type $j$. $\mathrm{T}$ is the absolute temperature. The ionic strength in $\mathrm{mol} / \mathrm{m}^{3}$ is denoted by $\mathrm{I}$.

The plasma parameter measures the relative strength of the direct electrostatic interaction between two ions to the collective interaction in the ionic cloud. In accordance with the method of Bogoliubov, this parameter is assumed to be small in comparison to unity, i.e. small ionic strengths. The Bjerrum parameter measures the electrostatic energy at contact relative to the kinetic energy $(k T)$, i.e. the tendency to form ion pairs as in the much more qualitative original theory of Bjerrum. ${ }^{27}$

The solvent-averaged pair potential between two ions assumed by Schmitz has two compo-

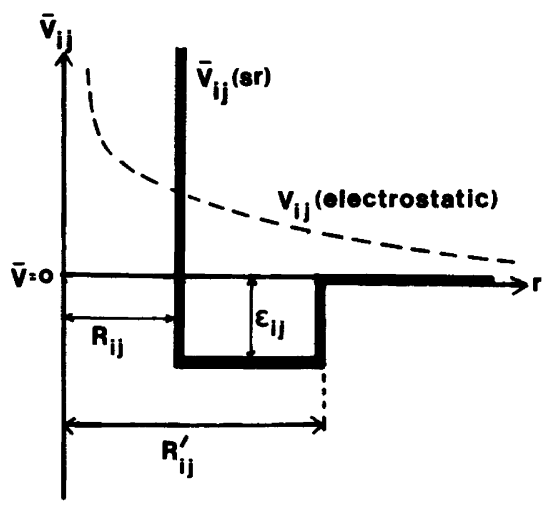

Fig. 1. Solvent averaged potential assumed by Schmitz. The potential is composed by a longrange electrostatic part and a short-range hard sphere/square well part.

nents, see Fig. 1. The long-range or electrostatic component is a simple Coulomb-potential:

$\bar{V}_{\mathrm{ij}}(r)=\frac{z_{\mathrm{i}} z_{\mathrm{j}} e_{\mathrm{o}}^{2}}{4 \pi \cdot \varepsilon \cdot r}$

The short-range (sr) potential is a hard-sphere interaction with a square-well attractive potential of depth $\varepsilon_{\mathrm{ij}}$. Such an attractive potential has proved useful to explain for example negative deviations from the limiting law for certain quaternary ammonium halides, see Ref. $10 \mathrm{pp}$. 418-421. Also, the alkali nitrates lie too close to the limiting law at higher concentrations than expected from reasonable distances of contact. If $R_{\mathrm{ij}}^{\prime}$ (see Fig. 1) is put equal to $2 R_{\mathrm{ij}}$, the attraction energy depths are approximately equal to the energy minima found for noble gases. Furthermore, in the case of nitrates one observes an increase in $\varepsilon_{12}$ with an increase in number of electrons in the cation. Therefore, the attractive forces are likely to be London-van der Waals forces.

Bogoliubov expansion to the first order in the plasma parameters $\mu_{\mathrm{ij}}$ leads to the Debye-Hückel limiting law (DHLL). We shall only consider here the corrections to DHLL from $O\left(\mu^{2}\right)$ terms, although Schmitz also carried out corrections of $O\left(\mu^{3}\right)$. (For transport phenomena in dense gases, a similar expansion procedure of Bogoliubov is known to diverge beyond the first correction terms because of the neglect of recollisions 
between molecules, ${ }^{28}$ but equilibrium phenomena are probably not sensitive to such recollisions, so the expansion may work to higher approximations here). Unfortunately, $O\left(\mu^{2}\right)$ limits us to $0.01 \mathrm{~mol} / \mathrm{dm}^{3}$ for $1: 1$ salts and 0.001 $\mathrm{mol} / \mathrm{dm}^{3}$ for $2: 2$ salts, but the Bjerrum parameters may be arbitrarily large.

In the simplest case, we have a cation 1 and an anion 2 of the same size (RPM) and with numerically identical charges $\left(z_{1}=-z_{2}=z\right.$, symmetric electrolyte). We then have:

$R_{11}=R_{22}=R_{12}=a$

(contact distance)

$\xi_{12}=-\xi_{11}=-\xi_{22}=b$

(positive Bjerrum parameter)

If we further assume the attractive potentials equal, we have:

$\varepsilon_{11}=\varepsilon_{22}=\varepsilon_{12}=\bar{\varepsilon}$

The expression for the osmotic pressure in that case becomes:

$\Pi=2 \cdot c_{\mathrm{s}} \cdot R T\left\{1-\frac{1}{6} \mu-\right.$

$\left.\frac{1}{2} \mu^{2}\left[b^{-3}(1+\tau) k_{\mathrm{o}}(b)-\left(b^{\prime}\right)^{-3} \tau k_{\mathrm{o}}\left(b^{\prime}\right)\right]\right\}$

The salt concentration $\left(\mathrm{mol} / \mathrm{m}^{3}\right)$ is $c_{\mathrm{s}} . \tau$ is a transformed attractive potential given by

$\tau=\exp (\bar{\varepsilon} / k T)-1$

and $k_{\mathrm{o}}$ is a complicated function of the Bjerrum parameter given by the following power series:

$$
\begin{aligned}
& k_{\mathrm{o}}(b)=-\frac{1}{3}-\frac{1}{2} b^{2}+ \\
& \sum_{\mathrm{m} \geq 2} \frac{b^{2 \mathrm{~m}}}{(2 \mathrm{~m}) !(2 \mathrm{~m}-3)}
\end{aligned}
$$

Finally, the parameter $b^{\prime}$ in eqn. (9) is defined as $b$ in eqn. (7), but with $R_{\mathrm{ij}}^{\prime}$ in lieu of $R_{\mathrm{ij}}$ in eqn. (3).

In case of simple hard spheres and $b$ not greater than 2 (where $k_{\mathrm{o}} \cong-\frac{1}{3}-\frac{1}{2} b^{2}$ ) we obtain the simple expression:

$$
\begin{aligned}
& \Pi=2 \cdot c_{\mathrm{s}} \cdot R T \times \\
& \left\{1-\frac{z^{2} e_{\mathrm{o}}^{2} \cdot \kappa}{24 \pi \cdot \varepsilon k T}\left(1-\frac{3}{2} \kappa a\right)+c_{\mathrm{s}} \cdot \frac{4 \pi N_{\mathrm{o}}}{3} a^{3}\right\}
\end{aligned}
$$

The last term in eqn. (12) is clearly an excluded volume term. The other terms are identical to the normal (extended) DH-expression for the osmotic pressure of a symmetric electrolyte, when this expression is power expanded to second order in $\kappa \cdot a$, thus matching the excluded volume term proportional to $c_{s}$. (By extended DH-theory we mean the DH theory taking into account that ions are not point charges, but have a certain contact distance $=a$ ).

For Bjerrum parameters greater than 2 , we have great deviations from this simple picture. For an extreme value of $b(b \geq 15)$, we have $k_{\mathrm{o}}(b)=\exp (b) / 2 b$ and the osmotic pressure becomes:

$$
\Pi=2 \cdot c_{\mathrm{s}} \cdot R T\left\{1-\frac{z^{2} \cdot e_{\mathrm{o}}^{2}}{24 \pi \varepsilon k T} \cdot \kappa-2 \pi \cdot c_{\mathrm{s}} N_{\mathrm{o}} a^{3} \cdot e^{b} / b\right\}
$$

It is obvious, that large Bjerrum parameters (strong ion pair formation) pull in the opposite direction of excluded volume effects, and the Bjerrum effects are able to more than cancel the excluded volumes. However, the requirements $b \gg 1$ and $\mu \ll 1$ together mean, that the ionic strength has to be extremely small. Very great values of $b$ are found for 2:2 and higher charged electrolytes in solvents with low $\varepsilon$. For 1:1, 2:1 and $1: 2$ electrolytes, $b$ is mostly less than 2 in aqueous solutions.

A general expression for the osmotic pressure in many-ion solutions with ions of different radii has also been derived by Schmitz (with $\varepsilon_{i j}=0$ ):

$$
\begin{aligned}
& \Pi / R T=\sum_{\mathrm{i}} c_{\mathrm{i}}\left\{1-\frac{z_{\mathrm{i}}^{2} \kappa \cdot e_{\mathrm{o}}^{2}}{24 \pi \varepsilon k T}-\right. \\
& \frac{\pi}{3} N_{\mathrm{o}} \sum_{\mathrm{j}} c_{\mathrm{j}} \cdot l_{\mathrm{ij}}^{3} \ln \left(\kappa\left|l_{\mathrm{ij}}\right|\right)- \\
& \left.2 \pi N_{\mathrm{o}} \sum_{\mathrm{j}} c_{\mathrm{j}}\left\{R_{\mathrm{ij}}^{3} L\left(l_{\mathrm{ij}} / R_{\mathrm{ij}}\right)-l_{\mathrm{ij}}^{3} / 12\right\}\right\}
\end{aligned}
$$


The concentration $\left(\mathrm{mol} / \mathrm{m}^{3}\right)$ of ion No. $\mathrm{i}$ is $c_{\mathrm{i}}$, and the distances (positive and negative) $l_{\mathrm{ij}}$ are defined as:

$l_{\mathrm{ij}}=-\frac{z_{\mathrm{i}} z_{\mathrm{j}} e_{\mathrm{o}}^{2}}{4 \pi \varepsilon k T}=R_{\mathrm{ij}} \cdot \xi_{\mathrm{ij}}$

The numerical value of $l_{\mathrm{ij}}$ is the distance between ion types No. $i$ and No. $j$, where the electrostatic energy is equal to $k T$. The function $L(\xi)$ in eqn. (14) is a complicated function with the expansion:

$$
\begin{aligned}
& L(\xi)=-\frac{1}{3}-\frac{1}{2} \xi-\frac{1}{2} \xi^{2}+ \\
& \frac{1}{6} \xi^{3} \ln |\xi|-\frac{1}{6} \xi^{3} \cdot(C+\ln 3)+K(\xi) \\
& K(\xi)=\sum_{m \geq 4} \frac{\xi^{m}}{m !(m-3)}
\end{aligned}
$$

$C=0.5772 \ldots=$ Euler's constant

Eqn. (14) can be rewritten in the following way:

$$
\begin{aligned}
& \Pi / R T=\sum_{\mathrm{i}} c_{\mathrm{i}}-\frac{2}{3} A_{\mathrm{DH}} \cdot I^{3 / 2}-\frac{1}{2} \sum_{\mathrm{i}} \sum_{\mathrm{j}} c_{\mathrm{i}} c_{\mathrm{j}} . \\
& v_{\mathrm{ij}}\left\{\frac{\xi_{\mathrm{ij}}^{3}}{2} \ln \left(\kappa \cdot R_{\mathrm{ij}}\right)\right\}+\frac{1}{2} \sum_{\mathrm{i}} \sum_{\mathrm{j}} c_{\mathrm{i}} c_{\mathrm{j}} \cdot v_{\mathrm{ij}} \cdot \Xi_{\mathrm{ij}}
\end{aligned}
$$

The Debye-Hückel constant is given by:

$$
A_{\mathrm{DH}}=\frac{F^{3} \sqrt{2}}{8 \pi N_{\mathrm{o}}(R T \varepsilon)^{3 / 2}}
$$

The excluded volume between one mole of ion pairs is:

$v_{\mathrm{ij}}=\frac{4 \pi}{3} R_{\mathrm{ij}}^{3} \cdot N_{\mathrm{o}}$

Finally, we have introduced the following functions of the Bjerrum parameters:

$$
\begin{aligned}
& \Xi_{\mathrm{ij}}=1+\frac{3}{2} \cdot \xi_{\mathrm{ij}}+\frac{3}{2} \cdot \xi_{\mathrm{ij}}^{2}+ \\
& \frac{1}{2} \cdot \xi_{\mathrm{ij}}^{3}\left(\mathrm{C}+\ln 3+\frac{1}{2}-2 \ln \left|\xi_{\mathrm{ij}}\right|\right)-3 \cdot K\left(\xi_{\mathrm{ij}}\right)
\end{aligned}
$$

One new feature in eqn. (19) is the appearance of terms of order $c^{2} \ln c$. Such terms are well known also in the Bogoliubov expansions for transport phenomena in dense gases. ${ }^{28}$ The terms cancel out for symmetric electrolytes with cations and anions of the same size. The logarithmic terms can be omitted for small Bjerrum parameters, where $O\left(\xi^{3}\right)$ and above are neglected. Neglecting $O(\xi)$ and above, we obtain:

$$
\Pi / R T \cong \sum_{\mathrm{i}} c_{\mathrm{i}}-\frac{2}{3} A \mathrm{DH} \cdot I^{3 / 2}+\underset{2}{2} \sum_{\mathrm{i}} \sum_{\mathrm{j}} c_{\mathrm{i}} c_{\mathrm{j}} v_{\mathrm{ij}}
$$

This equation has exactly the same form as the one considered by one of us (TSS) in a previous paper from more intuitive considerations, see Ref. 24 eqns. (16)-(21). It was there called Debye-Hückel Excluded Volume model, but a more proper name would be Limiting LawExcluded Volume (LLEV) model.

The problem with the LLEV-model is, that as soon as the excluded volume is felt, there will also be contributions to the $c^{2}$ term from the deviations from the limiting law for the electrostatic part of the osmotic pressure, or rather from the mixed electrostatic/excluded volume part, see eqn. (19). Neglecting those contributions, we get all too great contact distances, $c f$. Ref. 24, Table 1 and Table 2.

The change in osmotic pressure is related to the change in solvent activity by eqn. (24). ( $v_{\mathrm{o}}$ partial molar volume of solvent).

$$
R T \operatorname{dln} a_{\mathrm{o}}+v_{\mathrm{o}} \mathrm{d} \Pi=0
$$

From Gibbs-Duhem's equation and the approximation $c_{0} v_{0} \cong 1$, we readily derive for a single electrolyte:

$$
v \cdot c_{\mathrm{s}} \cdot\left(\frac{\mathrm{d} \ln y_{ \pm}}{\mathrm{d} c_{\mathrm{s}}}\right)_{c_{\mathrm{s}}} \cong\left(\frac{\mathrm{d}(\Pi / R T)}{\mathrm{d} c_{\mathrm{s}}}\right)_{c_{\mathrm{s}}}-v
$$

Here, $y_{ \pm}$is the mean ionic, molar activity coefficient for the electrolyte. Thus, to find this activity coefficient as a function of concentration we have to derive the expression for the osmotic pressure with respect to salt concentration, divide by the salt concentration and integrate with respect to salt concentration from $c_{\mathrm{s}}=0$ to $c_{\mathrm{s}}$. The calculations are tedious, but from eqns. (19) and (25) we obtain: 
$\ln y_{ \pm}=-\left|z_{1} z_{2}\right| A_{\mathrm{DH}} \sqrt{I}$

$$
\begin{aligned}
& -\frac{c_{\mathrm{s}}}{4} \sum_{\mathrm{i}=1}^{2} \sum_{\mathrm{j}=1}^{2}\left(\frac{v_{\mathrm{i}} v_{\mathrm{j}}}{v}\right) \cdot v_{\mathrm{ij}} \times \\
& \left\{\xi_{\mathrm{ij}}^{3}\left[\ln \left(R_{\mathrm{ij}}^{2} \mathrm{k}^{2}\right)-R_{\mathrm{ij}}^{2} \mathrm{k}^{2}\right]\right\} \\
& +c_{\mathrm{s}} \sum_{\mathrm{i}=1}^{2} \sum_{\mathrm{j}=1}^{2}\left(\frac{v_{\mathrm{i}} v_{\mathrm{j}}}{v}\right) v_{\mathrm{ij}} \cdot \chi_{\mathrm{ij}}
\end{aligned}
$$

We have introduced the stoichiometric coefficients in the salt, $v_{1}$ for the cation, $v_{2}$ for the anion $\left(v=v_{1}+v_{2}\right)$. Furthermore, we have introduced:

$$
\begin{aligned}
& \chi_{\mathrm{ij}}=\Xi_{\mathrm{ij}}-\frac{1}{8} \cdot \xi_{\mathrm{ij}}^{3}=1+\frac{3}{2} \xi_{\mathrm{ij}}+\frac{3}{2} \xi_{\mathrm{ij}}^{2} \\
& +\frac{1}{2} \xi_{\mathrm{ij}}^{3}\left\{C+\ln 3+\frac{1}{4}-2 \ln \left|\xi_{\mathrm{ij}}\right|\right\}-3 K\left(\xi_{\mathrm{ij}}\right)
\end{aligned}
$$

Eqn. (26) may be recast into a more useful form by expressing all Bjerrum parameters $\xi_{\mathrm{ij}}$ in terms of a single (positive) parameter:

$$
b=\xi_{12}>0
$$

We obtain up to $O\left(b^{3}\right)$ and up to $O\left(c_{\mathrm{s}}\right)$ the formula (29) given below as Scheme 1.

Eqn. (29) has a convenient form for exhibiting clearly the effect of various assumptions. If we consider a general asymmetric electrolyte, but with equal ionic radii, viz.

$R_{11}=R_{12}=R_{22}=a$ and $v_{11}=v_{12}=v_{22}=\frac{4 \pi}{3} a^{3} \cdot N_{\mathrm{o}}$

then the term linear in $b$ disappears, but not the $c_{\mathrm{s}} \ln (a \kappa)$ term. However, the logarithmic term may be neglected, if one neglects terms of $\mathrm{O}\left(b^{3}\right)$. If we furthermore assume the electrolyte to be symmetric, we have:

$$
v_{1}=v_{2}=1 ; v=2 ; z_{1}=-z_{2}=z
$$

Then, all the $b^{3}$ terms become identical to zero, and the $c_{\mathrm{s}} \ln (a \kappa)$ term vanishes, too. Introducing

$b^{2}=\frac{z^{4} \cdot e_{0}^{4}}{(4 \pi \varepsilon k T a)^{2}}$

we obtain:

$$
\begin{aligned}
& \ln y_{ \pm} \cong-z^{2} A_{\mathrm{DH}} \sqrt{I}+\frac{z^{2} \cdot \kappa^{2} e_{\mathrm{o}}^{2}}{8 \pi \varepsilon k T} \cdot a+ \\
& \frac{8 \pi}{3} \cdot a^{3} \cdot N_{\mathrm{o}} \cdot c_{\mathrm{s}}+O\left(b^{4}\right)
\end{aligned}
$$

This equation could have been derived in a much simpler way from eqns. (12) and (25). Eqn. (33) is identical to the Debye-Hückel Excluded Volume expression (DHEV), since we have:

$$
\begin{aligned}
& \ln y_{ \pm}(\mathrm{DH})=\frac{-\left|z_{1} z_{2}\right| A_{\mathrm{DH}} \cdot \sqrt{I}}{1+\kappa a}= \\
& -\left|z_{1} z_{2}\right| A_{D H} \sqrt{I}+\left|z_{1} z_{2}\right| \cdot A_{\mathrm{DH}} \cdot \kappa a \cdot \sqrt{I}+\mathrm{O}\left(I^{3 / 2}\right)
\end{aligned}
$$

One sees immediately, that:

$\left|z_{1} z_{2}\right| A_{\mathrm{DH}} \cdot \kappa \mathrm{a} \cdot \sqrt{I}=\frac{z_{1} z_{2} \cdot e_{\mathrm{o}}^{2} \cdot a \cdot \kappa^{2}}{8 \pi \varepsilon k T}$

No real electrolyte will have ionic radii, which are exactly equal, however. Thus, even if we restrict ourselves to $O\left(c_{\mathrm{s}}\right)$ and $O\left(b^{3}\right)$, the DHEV expres-

$$
\begin{aligned}
& \ln y_{ \pm} \cong-\left|z_{1} z_{2}\right| A_{\mathrm{DH}} \sqrt{I+\frac{1}{2}}\left(\frac{v_{1} v_{2}}{v}\right) c_{\mathrm{s}} \cdot b^{3}\left\{2 v_{12} \ln \left(R_{12} \kappa\right)-\left(\frac{v_{2}}{v_{1}}\right)^{2} \cdot\left(\frac{R_{12}}{R_{11}}\right)^{3} v_{11} \ln \left(R_{11} \kappa\right)\right. \\
& \left.-\left(\frac{v_{1}}{v_{2}}\right)^{2} \cdot\left(\frac{R_{12}}{R_{22}}\right)^{3} \cdot v_{22} \ln \left(R_{22} \kappa\right)\right\}+\left(\frac{v_{1} v_{2}}{v}\right) c_{\mathrm{s}} \cdot\left\{\left[2 v_{12}+v_{11}+v_{22}\right]+b \cdot\left[2 v_{12}-\left(\frac{R_{12}}{R_{11}}\right) \cdot v_{11}-\left(\frac{R_{12}}{R_{22}}\right) \cdot v_{22}\right]\right. \\
& +\frac{3}{2} b^{2} \cdot\left[2 v_{12}+\left(\frac{v_{2}}{v_{1}}\right) \cdot\left(\frac{R_{12}}{R_{11}}\right)^{2} \cdot v_{11}+\left(\frac{v_{1}}{v_{2}}\right) \cdot\left(\frac{R_{12}}{R_{22}}\right)^{2} \cdot v_{22}\right] \\
& +0.9629 \cdot b^{3} \cdot\left[2 v_{12}-\left(\frac{v_{2}}{v_{1}}\right)^{2} \cdot\left(\frac{R_{12}}{R_{11}}\right)^{3} \cdot v_{11}-\left(\frac{v_{1}}{v_{2}}\right)^{2} \cdot\left(\frac{R_{12}}{R_{22}}\right)^{3} \cdot v_{22}\right] \\
& \left.-b^{3} \cdot\left[2 v_{12} \ln b-\left(\frac{v_{2}}{v_{1}}\right)^{2} \cdot\left(\frac{R_{12}}{R_{11}}\right)^{3} \cdot \ln \left(\frac{v_{2}}{v_{1}} \cdot b\right)^{2} \cdot v_{11}-\left(\frac{v_{1}}{v_{2}}\right)^{2} \cdot\left(\frac{R_{12}}{R_{22}}\right)^{3} \cdot \ln \left(\frac{v_{1}}{v_{2}} b\right) \cdot v_{22}\right]\right\}
\end{aligned}
$$

\section{Scheme 1.}


sion (33) will not be perfect, neither for symmetric nor for asymmetric electrolytes, since the term linear in $b$ has been omitted.

For an arbitrary electrolyte, the term linear in $b$ in eqn. (29) is readily reformulated:

Linear term in $b$ in eqn. (29) $=$

$-\frac{2}{3}\left(\frac{v_{1} \cdot v_{2}}{v^{2}}\right) \cdot \kappa^{2} \cdot\left(R_{1}-R_{2}\right)^{2}$

Thus, the linear term contributes negatively to the $O\left(c_{\mathrm{s}}\right)$ term, and it depends on the square of the difference between the ionic radii $\left(R_{1}-R_{2}\right)$. This feature is unknown in the Debye-Hückel theory as usually formulated, but it might be gained from an extended Debye-Hückel theory, if the Debye-Hückel charging p:ocedure is carried out in a more careful manner than usual.

As a final comment in this section, we see from eqns. (19) and (26), that "excluded volume" terms in the osmotic pressure are manifest as "specific interaction" terms of order $c_{\mathrm{s}}$ in the formula for $\ln y_{ \pm}$. Obviously, there is no such thing as the Brønsted principle, excluding "specific interaction" between ions of like signs. All excluded volumes (cation-cation, cation-anion, anion-anion) should be accounted for according to the rigorous treatment of Schmitz.

\section{MEAN SPHERICAL APPROXIMATION (MSA) MODEL}

The MSA-model for general electrolyte mixtures has been summarized in papers of Blum et $a l .{ }^{29,30}$ Like other recent approaches based on the direct correlation function, the MSA-theory seems to neglect the problems in connection with the Bjerrum parameters. However, in contrast to the Bogoliubov-expansion described in section 1, the more "modern" theories (MSA and HNC) seem to work up well to $1 \mathrm{~mol} / \mathrm{dm}^{3}$ or more, when compared to Monte Carlo calculations on the "primitive model" (dielectric continuum assumption). Especially the MSA-theory yields very simple formulae, which bear strong resemblance to the DHEV-theory. We just have to generalize the expression (4) for the DH-parameter $(\kappa)$ into another inverse shielding length $(\Gamma)$. In Refs. 29 and 30 , it is $2 \Gamma$ which corresponds to $\kappa$, but we have made a slight reformulation here.
In the MSA-theory, the mean ionic, molar activity coefficient for a general electrolyte is given by:

$\ln y_{ \pm}(\mathrm{MSA})=\mathrm{g}(\Gamma)+2 \cdot \beta_{2} \cdot \mathrm{N}_{\mathrm{o}} \mathrm{c}_{\mathrm{s}}$

The electrostatic/contact-distance contribution is given by the function:

$$
\mathrm{g}(\Gamma)=-\frac{\kappa^{2} \Gamma}{\alpha \cdot \mathrm{c}_{\mathrm{s}}} \sum_{\mathrm{i}} \frac{v_{\mathrm{i}} \mathrm{z}_{\mathrm{i}}^{2}}{1+\beta \cdot \Gamma \cdot \mathrm{R}_{\mathrm{ii}}}
$$

Summations are over the ions in the salt, and $v_{\mathrm{i}}$ and $\mathbf{R}_{\mathrm{ii}}$ are stoichiometric coefficients and ionic diameters as in section 1 . The parameters $\alpha$ and $\beta$ will be fixed later by comparison to DebyeHückel theory. The parameter in the "specific interaction" term of eqn. (37) is given by: ${ }^{29}$

$$
\begin{aligned}
& \beta_{2}=\frac{\pi}{2}\left(\frac{\xi_{3}}{3}+\frac{\xi_{1} \xi_{2}}{\xi_{\mathrm{o}}}\right) \\
& \xi_{\mathrm{n}} \equiv \sum_{\mathrm{i}} v_{\mathrm{i}} \cdot R_{\mathrm{ii}}^{\mathrm{n}}
\end{aligned}
$$

From eqn. (39) we obtain:

$$
\begin{aligned}
& \beta_{2}=\frac{1}{2}\left(\frac{4 \pi}{3} R_{11}^{3}\right) \cdot(\mathrm{r})^{-3} \cdot v \cdot\left\{\mathrm{r}, v_{1} / v, v_{2} / v\right\} \\
& r \equiv R_{11} / R_{22} \\
& \left\{\mathrm{r}, v_{1} / v, v_{2} / v\right\} \equiv\left[\frac{1}{4} \cdot \frac{v_{1}}{v}+\frac{3}{4} \cdot\left(\frac{v_{1}}{v}\right)^{2}\right] \cdot r^{3}+ \\
& \frac{3}{4}\left(\frac{v_{1}}{v}\right)\left(\frac{v_{2}}{v}\right) r^{2}+\frac{3}{4}\left(\frac{v_{1}}{v}\right)\left(\frac{v_{2}}{v}\right) r+\left[\frac{1}{4} \cdot \frac{v_{2}}{v}+\frac{3}{4}\left(\frac{v_{2}}{v}\right)^{2}\right]
\end{aligned}
$$

Especially for symmetric electrolytes $(v=2$, $v_{1}=v_{2}=1$ ) we have:

$$
\left\{r, \frac{1}{2}, \frac{1}{2}\right\}=\frac{1}{16} \cdot\left(5 r^{3}+3 r^{2}+3 r+5\right)
$$

On the other hand, from eqn. (26) with $\chi_{i j}=1$, we get "excluded volume" terms of the form:

$$
\beta_{2}=\frac{v}{2} \cdot c_{\mathrm{s}} \cdot \sum_{\mathrm{i}} \sum_{\mathrm{j}}\left(\frac{v_{\mathrm{i}}}{v}\right)\left(\frac{v_{\mathrm{j}}}{v}\right)\left(4 \pi R_{\mathrm{ij}}^{3} / 3\right)
$$

This may be recast into the form:

$$
\beta_{2}=\frac{v}{2}\left(4 \pi \cdot R_{11}^{3} / 3\right)(r)^{-3} \cdot\left[r, v_{1} / v, v_{2} / v\right]
$$


We have defined:

$\left[\mathrm{r}, v_{1} / v, v_{2} / v\right] \equiv\left[\frac{1}{4}\left(\frac{v_{1}}{v}\right)\left(\frac{v_{2}}{v}\right)+\left(\frac{v_{1}}{v}\right)^{2}\right] \cdot r^{3}$

$+\frac{3}{4}\left(\frac{v_{1}}{v}\right)\left(\frac{v_{2}}{v}\right) r^{2}+\frac{3}{4}\left(\frac{v_{1}}{v}\right)\left(\frac{v_{2}}{v}\right) r$

$+\left[\frac{1}{4}\left(\frac{v_{1}}{v}\right)\left(\frac{v_{2}}{v}\right)+\left(\frac{v_{2}}{v}\right)^{2}\right]$

$=\left(\frac{v_{1}}{v}\right)^{2} \cdot r^{3}+\frac{1}{4}\left(\frac{v_{1}}{v}\right)\left(\frac{v_{2}}{v}\right)(r+1)^{3}+\left(\frac{v_{2}}{v}\right)^{2}$

In general, we have

$$
\left\{r, v_{1} / v, v_{2} / v\right\} \neq\left[\mathrm{r}, v_{1} / v, v_{2} / v\right]
$$

but the two expressions coincide for symmetric electrolytes with the function of $r$ given in eqn. (44). Most probably, Ref. 29 (from which eqns. (39) and (40) come) is slightly in error, and the formulae (39) and (40) are only valid in the symmetric case. Actually, only experimental activity coefficients of 1:1 electrolytes were studied in Ref. 29. The expression (46) follows also directly for an uncharged, "hard sphere solution" with two types of spheres, by making the usual analogy between the osmotic pressure and the pressure of a mixed hard sphere gas, see Ref. 24. This analogy is rigorously based in statistical mechanics according to Mayer. ${ }^{31}$ In the following, we shall take the excluded volume contribution of the MSA-theory as eqns. (46) and (47).

We now return to the $\mathrm{g}(I)$-contribution to lny $y_{ \pm}$. The generalized inverse shielding length $(\Gamma)$ has to be found as a solution to the following equation:

$$
\gamma \equiv \Gamma / \kappa=\frac{1}{\sqrt{\sum_{\mathrm{i}} v_{\mathrm{i}} z_{\mathrm{i}}^{2}}} \cdot \sqrt{\sum_{\mathrm{i}} \frac{v_{\mathrm{i}} z_{\mathrm{i}}^{2}}{\left(1+\beta \cdot \gamma\left(\kappa \cdot R_{\mathrm{ii}}\right)\right)^{2}}}
$$

Now, we define:

$\mathrm{x}=\beta R_{11} \kappa ; \quad \mathrm{s}_{\mathrm{i}}=R_{\mathrm{ii}} / R_{11}$

From eqns. (38) and (49) we obtain:

$$
\begin{aligned}
& \mathrm{g}(\gamma, x)=-\frac{\kappa^{3}}{\eta \mathrm{c}_{\mathrm{s}}} \cdot \gamma \cdot \sum_{\mathrm{i}} \frac{v_{\mathrm{i}} \mathrm{z}_{\mathrm{i}}^{2}}{1+\gamma \cdot s_{\mathrm{i}} \cdot x} \\
& \gamma(x)=\frac{1}{\sqrt{\sum_{\mathrm{i}} v_{\mathrm{i}} \cdot z_{\mathrm{i}}^{2}}} \cdot \sqrt{\sum_{\mathrm{i}} \frac{v_{\mathrm{i}} z_{\mathrm{i}}^{2}}{\left(1+\gamma \cdot s_{\mathrm{i}} \cdot x\right)^{2}}}
\end{aligned}
$$

When the terms $\gamma \cdot s_{\mathrm{i}} \cdot x$ are small compared to unity, the denominator in eqn. (52) can be replaced by its McLaurin expansion from $x=0$. Retaining terms up to $x^{2}$, we obtain the following quadratic equation for $\gamma$ as a function of $x$ :

$\left[3 S_{2} x^{2}-1\right] \gamma^{2}-2 S_{1} \cdot x \cdot \gamma+1=0$

$S_{1}=\frac{\sum_{v_{\mathrm{i}} z_{\mathrm{i}}^{2} s_{\mathrm{i}}}}{\sum_{v_{\mathrm{i}} z_{\mathrm{i}}^{2}}}$

$S_{2}=\frac{\sum_{v_{\mathrm{i}} z_{\mathrm{i}}^{2} s_{\mathrm{i}}^{2}}}{\sum_{v_{\mathrm{i}} z_{\mathrm{i}}^{2}}}$

The two roots converge towards +1 and -1 as $x \rightarrow 0$, and the positive root should be chosen:

$\gamma \cong \frac{\sqrt{1+\left(S_{1}^{2}-3 S_{2}\right) x^{2}}-S_{1} x}{1-3 S_{2} \cdot x^{2}}$

In the "restricted primitive model", we have $S_{1}=S_{2}=1$, and eqn. (57):

$\gamma \cong \frac{\sqrt{1-2 x^{2}}-x}{1-3 x^{2}}(\mathrm{RPM})$

Note, that eqn. (57) is valid for asymmetric as well as symmetric electrolytes, if only all ionic radii are equal.

In the RPM we have from eqn. (51):

$\mathrm{g}(\gamma, x)=-\frac{\kappa^{3} \cdot \gamma}{\eta \cdot c_{\mathrm{s}}} \cdot \frac{\sum_{v_{\mathrm{i}} z_{\mathrm{i}}^{2}}}{1+\gamma \cdot x} \quad(\mathrm{RPM})$

We use that (because of electroneutrality):

$I / c_{\mathrm{s}}=\frac{1}{2} \sum_{\mathrm{i}} v_{\mathrm{i}} z_{\mathrm{i}}^{2}=\frac{1}{2} v\left|z_{1} z_{2}\right|$

From eqns. (4), (20), (58) and (59) we obtain:

$\mathrm{g}(\gamma, \mathbf{x})=-A_{\mathrm{DH}} \cdot\left|z_{1} z_{2}\right| \frac{\gamma \sqrt{I}}{1+\gamma x}$

We have chosen

$\eta=8 \pi N_{\mathrm{o}} v \sum_{\mathrm{i}} v_{\mathrm{i}} z_{\mathrm{i}}^{2}$

in order to conform with the usual DHLL expression at low ionic strengths, where $\gamma=1$.

From eqn. (57) we obtain:

Acta Chem. Scand. A 38 (1984) No. 10 
$\frac{\gamma}{1+\gamma x} \cong \frac{\sqrt{1-2 x^{2}}-x}{1+x \sqrt{1-2 x^{2}}-4 x^{2}}$

The McLaurin series is found to be $\left(R_{11}=R_{22}=R_{12}=a\right)$ :

$$
\frac{\gamma}{1+\gamma x} \cong 1-2 x+6 x^{2}=1-2 \beta \cdot a \kappa+6 \cdot \beta^{2} \cdot(a \kappa)^{2}(
$$

In the Debye-Hückel approach we have similarly:

$\frac{1}{1+\kappa a} \cong 1-\kappa a+(\kappa a)^{2}$

Thus, by choosing

$$
\beta=\frac{1}{2}
$$

we have correspondence between the DHEV and the MSA-theory up to terms of $O\left(c_{\mathrm{s}}\right)$. The choice $\beta=\frac{1}{2}$ was indeed the one made in Refs. 29 and 30 . It should be noticed, that the limiting form for RPM [eqn. (62)] obtained from the MSA-theory of Blum et al. is somewhat different from the expression given originally by Waisman and Lebowitz. However, the McLaurin series of the two expressions are identical to the order $(\kappa a){ }^{2}$

\section{IONIC RADII FROM DHEV I AND DHEV II}

Having now certified, that the Debye-Hückel/ Excluded Volume theory can be proven on more rigorous foundation than the Debye-Hückel theory itself (at small Bjerrum parameters and with neglect of terms depending on differences on ionic radii!), we can move on and see if consistent contact distances can be calculated from data for $\ln y_{ \pm}$in sufficiently dilute solution.

Guggenheim et al. ${ }^{20,21}$ have made two-parameter fits to such data using the following formula for the molal activity coefficient:

$$
\begin{aligned}
& \ln \gamma_{ \pm}=-A_{\mathrm{DH}}^{\prime}\left|z_{1} z_{2}\right| \frac{\sqrt{I^{\prime}}}{1+B^{\prime} \cdot \stackrel{a}{\mathrm{G}}_{\mathrm{G}} \cdot \sqrt{I^{\prime}}} \\
& +\frac{4 v_{1} \cdot v_{2}}{v} \cdot \beta_{\mathrm{G}} \cdot m_{\mathrm{s}}
\end{aligned}
$$

$I^{\prime}$ is the ionic strength in $\mathrm{mol} / \mathrm{kg}$ solvent, and $m_{\mathrm{s}}$ the salt molality. The dashes refer to molal units for concentrations. The "contact distance" $\stackrel{a}{G}_{G}$ is in units of $0.1 \mathrm{~nm}$ (=Ångstrøm's). The chosen form of the "specific interaction" term corre- sponds to the Brønsted principle of no specific interaction between ions of like sign. In the following, we shall name DHEV I a DebyeHückel model without the Brønsted principle and DHEV II a DH-model with the Brønsted principle for the excluded volumes. The former will be the "correct" one for statistical-mechanical reasons, see sections 1 and 2 .

In the DHEV-models, the specific interaction term is also determined by the contact distance (a), so we must transform the two-parameter fits to one-parameter fits. Furthermore, expressing activity data in molal quantities is a bad habit in electrochemistry, which has survived from the early (pre-Debye-Hückelian) paper of Lewis and Randall. ${ }^{32}$ In statistical mechanics, concentration is fundamental, not molality, since concentration is measuring mean distance between dissolved, interacting ions.

At the low ionic strengths considered by Guggenheim et al. $\left(I \leq 0.01 \mathrm{~mol} / \mathrm{dm}^{3}\right)$, we may neglect differences between molal and molar activity coefficients, which are linked by eqn. (67):

$\mathrm{y}_{ \pm}=\gamma_{ \pm} \cdot d_{\mathrm{o}} \cdot\left(m_{\mathrm{s}} / c_{\mathrm{s}}\right)$

( $d_{\mathrm{o}}=$ density of pure solvent, $c_{\mathrm{s}}=$ molar salt concentration, $m_{\mathrm{s}}=$ molality of salt). Thus, we write instead of eqn. (66):

$$
\begin{aligned}
& \ln \mathrm{y}_{ \pm} \cong-A_{\mathrm{DH}}\left|\mathrm{z}_{1} \mathrm{z}_{2}\right| \frac{\sqrt{I}}{1+B_{\mathrm{DH}} \cdot \stackrel{\circ}{\mathrm{G}}_{\mathrm{G}} \cdot \sqrt{I}} \\
& +\frac{4 v_{1} v_{2}}{v} \cdot \beta_{\mathrm{G}} \cdot\left(\frac{c_{\mathrm{s}}}{d_{\mathrm{o}}}\right)
\end{aligned}
$$

Here, $A_{\mathrm{DH}}$ is the usual Debye-Hückel constant (see eqn. (20), but with an additional factor $\sqrt{1000}$, since I is in $\mathrm{mol} / \mathrm{dm}^{3}$ ). Furthermore, we have:

$$
B_{\mathrm{DH}}=B^{\prime} / \sqrt{d_{\mathrm{o}}}
$$

On the other hand, the DHEV-expression can be written:

$$
\begin{aligned}
& \ln \mathrm{y}_{ \pm}=-A_{\mathrm{DH}}\left|z_{1} z_{2}\right| \frac{\sqrt{I}}{1+B \cdot \stackrel{a}{a} \cdot \sqrt{I}}+ \\
& c_{\mathrm{s}} \cdot\left\{B_{11} \cdot\left(\frac{v_{1}^{2}}{v}\right) \delta+2 \cdot\left(\frac{v_{1} \cdot v_{2}}{v}\right) \cdot B_{12}+B_{22} \cdot\left(\frac{v_{2}^{2}}{v}\right) \delta\right\}
\end{aligned}
$$


We have for the parameter $\delta$ :

$$
\delta=\left\{\begin{array}{lll}
1 & \text { DHEV I } & \text { (No Brønsted assumption) } \\
0 & \text { DHEV II } & \text { (Brønsted assumption) }
\end{array}\right.
$$

The $B_{\mathrm{ij}}$-coefficients are excluded volumes defined as the $v_{\mathrm{ij}}$-coefficients in eqn. (21), but in $\mathrm{dm}^{3} / \mathrm{mol}$ :

$$
B_{\mathrm{ij}}=v_{\mathrm{ij}} \cdot 1000
$$

Now, we want to make the two expressions (68) and (70) identical up to terms of order $O\left(c_{\mathrm{s}}\right)$. Therefore, we Taylor-expand the Debye-Hückel denominator in both expressions, and furthermore we express all the $B_{\mathrm{ij}}$ 's in terms of $\dot{a}$ and $r=R_{11} / R_{22}$ [see eqn. (42)]. By identification we obtain a cubic equation in $d$ [Scheme 2, eqn. (73)]. For $1: 1$ electrolytes at $25^{\circ} \mathrm{C}\left(\mathrm{A}_{\mathrm{DH}}=1.1779\right.$ $\left.\mathrm{dm}^{3 / 2} \mathrm{~mol}^{-\frac{1}{2}}, \mathrm{~B}_{\mathrm{DH}}=0.3291 \mathrm{dm}^{3 / 2} \cdot \mathrm{mol}^{\frac{1}{2}} \cdot(0.1 \mathrm{~nm})^{-1}\right)$ we have eqn. (74), for $2: 1$ electrolytes $\left(\mathrm{CaCl}_{2}\right)$ eqn. (75) and for 1:2 electrolytes $\left(\mathrm{Na}_{2} \mathrm{SO}_{4}\right)$ eqn. (76). (We have also used that $d_{\mathrm{o}}=0.997 \mathrm{~kg} / \mathrm{dm}^{3}$ for water at $25^{\circ} \mathrm{C}$.)

Therefore, from the tabulations of $\beta_{\mathrm{G}}$ and $B^{\prime} \cdot \check{a}_{\mathrm{G}}$ at $25^{\circ} \mathrm{C}$ for various salts, we can calculate the contact distance $\stackrel{a}{a}$, if some assumption concerning the ratio of ionic radii $(r)$ is made. In the case of $a$ DHEV II $(\delta=0)$ we do not need any assumption. We shall use here the StokesEinstein assumption

$r=R_{11} / R_{22} \approx\left|\frac{z_{1}}{z_{2}}\right| \cdot \frac{\lambda_{2}^{0}}{\lambda_{1}^{\mathrm{o}}}$ where $\lambda_{\mathrm{i}}^{o}$ is the limiting equivalent conductivity for ion No. i. The Stokes-Einstein assumption is not expected to be very good, but the calculated values of $a$ are not very sensitive to the choice of the ratio $r$. (Indeed even to the choice of $\delta !$ ). For 1:1 salts, the resulting contact distances are tabulated in Table 1, whereas calculations for 2:1 and 1:2 salts have been collected in Table 2. (The cubic equation was solved on a HP 41C computer by the root seeking procedure for polynomials built into the mathematics module. In all cases, the cubic equation had only one real root).

From the tables 1,2 and 3, the following conclusions can be drawn:

(1) The values of $\dot{a}$ (DHEV II) are only slightly larger than $a$ (DHEV I). The greatest deviations $(0.3)$ are found for large values of $\stackrel{a}{ }$ and for 1:1 electrolytes. The reason for the small deviations is the dominance of the DH-denominator term over excluded volume terms.

(2) For electrolytes with ions normally assumed to be strongly hydrated $\left(\mathrm{Li}^{+}, \mathrm{Na}^{+}\right.$, $\mathrm{Mg}^{2+}, \mathrm{Ca}^{2+}, \mathrm{Sr}^{2+}, \mathrm{Ba}^{2+}, \mathrm{Mn}^{2+}, \mathrm{Fe}^{2+}, \mathrm{Co}^{2+}$, $\mathrm{Ni}^{2+}, \mathrm{Cu}^{2+}$ ) we find $a$ (DHEV I) $>R_{12}$ (crystal), where $R_{12}$ (crystal) is the sum of crystal ionic radii.

(3) The deviations between $\stackrel{a}{ }$ (DHEV I) and $R_{12}$ (crystal) are greater for doubly charged cations and for small singly charged cations (stronger hydration).

(4) The contact distance $a$ (DHEV I) for $\mathrm{KCl}$, $\mathrm{KBr}, \mathrm{KOH}$ is approximately the same as $R_{12}$ (crystal). Especially $\mathrm{KCl}$ seems to be an almost perfectly symmetric electrolyte with isoelectronic cation and anion. However, the symmetry is destroyed, because the $\mathrm{K}$-nucleus has two pro-

$$
\begin{aligned}
& {\left[\frac{8 \delta}{(r+1)^{3}} \cdot\left\{\frac{v_{1}^{2}}{v} \cdot r^{3}+\frac{v_{2}^{2}}{v}\right\}+\frac{2 v_{1} v_{2}}{v}\right]\left(4 \pi N_{\mathrm{o}} \cdot 10^{-27} / 3\right) \cdot \dot{a}^{3}} \\
& +A_{\mathrm{DH}} \cdot B_{\mathrm{DH}}\left(z_{1} z_{2}\right)^{2} \cdot(v / 2) \cdot \stackrel{\circ}{a}=\frac{4 v_{1} v_{2}}{v} \cdot\left(\frac{\beta_{\mathrm{G}}}{d_{\mathrm{o}}}\right)+\frac{v}{2} \cdot\left(z_{1} z_{2}\right)^{2} \cdot A_{\mathrm{DH}} \cdot \frac{B^{\prime} \dot{a}_{\mathrm{G}}}{\sqrt{d_{\mathrm{o}}}}
\end{aligned}
$$

$2.52 \cdot 10^{-3}\left\{1+4 \delta \frac{r^{3}+1}{(r+1)^{3}}\right\} \cdot \dot{a}^{3}+0.388 \cdot \dot{a}=2.01 \cdot \beta_{\mathrm{G}}+1.179 \mathrm{~B}^{\prime} \stackrel{\circ}{\mathrm{G}}_{\mathrm{G}} \quad\left(1: 1,25^{\circ} \mathrm{C}\right)$

$3.36 \cdot 10^{-3}\left\{1+2 \delta \frac{r^{3}+4}{(r+1)^{3}}\right\} \cdot \dot{a}^{3}+2.33 \cdot \stackrel{a}{a}=2.67 \cdot \beta_{\mathrm{G}}+7.07 \cdot B^{\prime} \stackrel{\circ}{\mathrm{G}}_{\mathrm{G}} \quad\left(2: 1,25^{\circ} \mathrm{C}\right)$

$3.36 \cdot 10^{-3}\left\{1+2 \cdot \delta \frac{4 r^{3}+1}{(r+1)^{3}}\right\} \cdot \dot{a}^{3}+2.33 \cdot \dot{a}=2.67 \cdot \beta_{\mathrm{G}}+7.07 \cdot B^{\prime} \cdot \stackrel{a}{\mathrm{G}} \quad\left(1: 2,25{ }^{\circ} \mathrm{C}\right)$

Scheme 2. 
Table 1. Contact distances (a) from DHEV I and DHEV II for $1: 1$ electrolytes at $25^{\circ} \mathrm{C}$ in water. $\left(B^{\prime} \cdot \dot{a}_{\mathrm{G}}=1 \mathrm{~kg}^{\frac{1}{2}} \cdot \mathrm{mol}^{\frac{1}{2}}\right.$ for all data).

\begin{tabular}{|c|c|c|c|c|}
\hline $\begin{array}{l}\text { Elec- } \\
\text { trolyte }\end{array}$ & $\beta_{\mathrm{G}}(\mathrm{kg} / \mathrm{mol})^{\mathrm{a}}$ & $r^{\mathrm{b}}$ & $\begin{array}{l}\stackrel{\therefore}{a}(0.1 \mathrm{~nm}) \\
\delta=1, \text { DHEV I }\end{array}$ & $\begin{array}{l}\stackrel{\therefore}{a}(0.1 \mathrm{~nm}) \\
\delta=0, \text { DHEV II }\end{array}$ \\
\hline $\mathrm{HCl}$ & 0.27 & (2) & 3.7 & 4.0 \\
\hline $\mathrm{HBr}$ & 0.33 & (2) & 3.9 & 4.2 \\
\hline HI & 0.36 & (2) & 4.0 & 4.4 \\
\hline $\mathrm{LiCl}$ & 0.22 & 2.0 & 3.5 & 3.8 \\
\hline $\mathrm{LiBr}$ & 0.26 & 2.0 & $3.6_{5}$ & 4.0 \\
\hline LiI & 0.35 & 2.0 & 3.9 & 4.3 \\
\hline $\mathrm{NaF}$ & 0.07 & (1.1) & 3.0 & 3.2 \\
\hline $\mathrm{NaCl}$ & 0.15 & 1.55 & 3.3 & 3.5 \\
\hline $\mathrm{NaBr}$ & 0.17 & 1.55 & 3.4 & 3.6 \\
\hline $\mathrm{NaI}$ & 0.21 & 1.55 & 3.5 & 3.8 \\
\hline $\mathrm{KF}$ & 0.13 & $(0.75)$ & $3.2_{5}$ & 3.4 \\
\hline $\mathrm{KCl}$ & 0.10 & 1.05 & $3.1_{5}$ & 3.3 \\
\hline $\mathrm{KBr}$ & 0.11 & 1.05 & 3.2 & 3.4 \\
\hline KI & 0.15 & 1.05 & 3.3 & 3.5 \\
\hline $\mathrm{RbCl}$ & 0.06 & 1.0 & 3.0 & $3.1_{5}$ \\
\hline $\mathrm{RbBr}$ & 0.05 & 1.0 & 3.0 & 3.1 \\
\hline RbI & 0.04 & 1.0 & 2.9 & 3.1 \\
\hline $\mathrm{CsCl}$ & 0.00 & 1.0 & 2.8 & 2.9 \\
\hline $\mathrm{CsBr}$ & 0.00 & 1.0 & 2.8 & 2.9 \\
\hline CsI & -0.01 & 1.0 & 2.7 & 2.8 \\
\hline $\mathrm{HNO}_{3}$ & 0.20 & (1.85) & 3.5 & 3.7 \\
\hline $\mathrm{LiNO}_{3}$ & 0.21 & 1.85 & 3.5 & 3.8 \\
\hline $\mathrm{NaNO}_{3}$ & 0.04 & 1.45 & 2.9 & 3.1 \\
\hline $\mathrm{KNO}_{3}$ & -0.11 & 1.0 & 2.3 & 2.4 \\
\hline $\mathrm{RbNO}_{3}$ & -0.14 & 0.9 & 2.2 & $2.2_{5}$ \\
\hline $\mathrm{CsNO}_{3}$ & -0.15 & 0.9 & 2.1 & 2.2 \\
\hline $\mathrm{AgNO}_{3}$ & -0.14 & 1.15 & 2.2 & $2.2_{5}$ \\
\hline $\mathrm{NH}_{4} \mathrm{Cl}$ & 0.10 & 1.0 & $3.1_{5}$ & 3.3 \\
\hline $\mathrm{NH}_{4} \mathrm{NO}_{3}$ & -0.10 & 1.0 & 2.4 & 2.4 \\
\hline $\mathrm{LiOH}$ & -0.21 & (1) & 1.9 & 1.9 \\
\hline $\mathrm{NaOH}$ & 0.06 & (1) & 3.0 & $3.1_{5}$ \\
\hline $\mathrm{KOH}$ & 0.13 & (1) & 3.3 & 3.4 \\
\hline $\mathrm{CsOH}$ & 0.35 & (1) & 4.0 & 4.3 \\
\hline $\mathrm{HClO}_{4}$ & 0.30 & $(1.75)$ & 3.8 & 4.1 \\
\hline $\mathrm{LiClO}_{4}$ & 0.34 & 1.75 & 3.9 & 4.3 \\
\hline $\mathrm{NaClO}_{4}$ & 0.13 & 1.3 & $3.2_{5}$ & 3.4 \\
\hline $\mathrm{NaClO}_{3}$ & 0.10 & 1.3 & $3.1_{5}$ & 3.3 \\
\hline $\mathrm{NaBrO}_{3}$ & 0.01 & 1.1 & 2.8 & 2.9 \\
\hline $\mathrm{KClO}_{3}$ & -0.04 & 0.9 & 2.6 & 2.7 \\
\hline $\mathrm{KBrO}_{3}$ & -0.07 & 0.75 & 2.5 & 2.6 \\
\hline
\end{tabular}

${ }^{a}$ From Table 30-1 in Ref. 33, where the data of Guggenheim are supplemented by some few others. ${ }^{b}$ Rounded values. Parenthesis indicates uncertain or invalid Stokes-Einstein hypothesis or lack of conductivity data.

tons more than the Cl-nucleus. Therefore, $\mathrm{K}^{+}$is smaller (radius $0.133 \mathrm{~nm})$ than $\mathrm{Cl}^{-}(0.181 \mathrm{~nm})$. Thus, if it is concluded that $\mathrm{K}^{+}$and $\mathrm{Cl}^{-}$are not hydrated, the two ions are dissimilar in size. This is inconsistent with the fact that they have almost identical limiting ionic conductivity. Most prob- ably, at least $\mathrm{K}^{+}$is somewhat hydrated, and $\stackrel{a}{a}$ (DHEV I) is too small due to attractive forces (see later). Another candidate for a symmetric electrolyte would be $\mathrm{KF}$, since the $\mathrm{K}^{+}$-ion and the $\mathrm{F}^{-}$ion have identical crystal ionic radii $(0.133 \mathrm{~nm})$. However, the ionic conductivity of $\mathrm{F}^{-}$

Acta Chem. Scand. A 38 (1984) No. 10 
Table 2. Contact distances (a) from DHEV I and DHEV II for 2:1 and 1:2 electrolytes at $25^{\circ} \mathrm{C}$ in water. (Data from Ref. 21).

\begin{tabular}{|c|c|c|c|c|c|}
\hline $\begin{array}{l}\text { Elec- } \\
\text { trolyte }\end{array}$ & $\begin{array}{l}(8 / 3) \beta_{\mathrm{G}} \\
\mathrm{kg} / \mathrm{mol}\end{array}$ & $\begin{array}{l}B^{\prime} ; \stackrel{a}{\mathrm{G}} \\
\mathrm{kg}^{\frac{1}{2}} \mathrm{~mol}^{\frac{1}{2}}\end{array}$ & $r^{b}$ & $\begin{array}{l}\stackrel{8}{a}(0.1 \mathrm{~nm}) \\
\delta=1, \text { DHEV I }\end{array}$ & $\begin{array}{l}\stackrel{8}{a}(0.1 \mathrm{~nm}) \\
\delta=0, \mathrm{DHEV} \text { II }\end{array}$ \\
\hline $\mathrm{MgCl}_{2}$ & 0.474 & 1.59 & 2.9 & 4.7 & 4.9 \\
\hline $\mathrm{MgBr}_{2}$ & 0.670 & 1.62 & 2.9 & 4.9 & 5.0 \\
\hline $\mathrm{CaCl}_{2}$ & 0.389 & 1.54 & 2.6 & 4.6 & 4.7 \\
\hline $\mathrm{CaBr}_{2}$ & 0.488 & 1.62 & 2.6 & 4.8 & 4.9 \\
\hline $\mathrm{SrCl}_{2}$ & 0.288 & 1.56 & 2.6 & 4.6 & 4.7 \\
\hline $\mathrm{SrBr}_{2}$ & 0.405 & 1.62 & 2.6 & 4.8 & 4.9 \\
\hline $\mathrm{BaCl}_{2}$ & 0.152 & 1.56 & 2.4 & $4.5_{5}$ & 4.7 \\
\hline $\mathrm{BaBr}_{2}$ & 0.322 & 1.56 & 2.5 & 4.6 & 4.7 \\
\hline $\mathrm{Mg}\left(\mathrm{NO}_{3}\right)_{2}$ & 0.479 & 1.53 & 2.7 & 4.6 & 4.7 \\
\hline $\mathrm{Ca}\left(\mathrm{NO}_{3}\right)_{2}$ & 0.120 & 1.39 & 2.4 & 4.1 & 4.2 \\
\hline $\mathrm{Sr}\left(\mathrm{NO}_{3}\right)_{2}$ & -0.099 & 1.39 & 2.4 & 4.0 & 4.1 \\
\hline $\mathrm{MnCl}_{2}$ & 0.341 & 1.56 & (2.8) & 4.6 & 4.7 \\
\hline $\mathrm{FeCl}_{2}$ & 0.373 & 1.56 & (2.8) & 4.6 & 4.7 \\
\hline $\mathrm{CoCl}_{2}$ & 0.433 & 1.56 & 2.8 & 4.6 & 4.8 \\
\hline $\mathrm{Co}\left(\mathrm{NO}_{3}\right)_{2}$ & 0.341 & 1.59 & 2.6 & 4.7 & 4.8 \\
\hline $\mathrm{NiCl}_{2}$ & 0.433 & 1.56 & (2.8) & 4.6 & 4.8 \\
\hline $\mathrm{CuCl}_{2}$ & 0.193 & 1.56 & 2.7 & $4.5_{5}$ & 4.7 \\
\hline $\mathrm{Cu}\left(\mathrm{NO}_{3}\right)_{2}$ & 0.281 & 1.53 & 2.5 & $4.5^{\circ}$ & 4.6 \\
\hline $\mathrm{Li}_{2} \mathrm{SO}_{4}$ & -0.150 & $1.41_{5}$ & 1.0 & 4.0 & 4.1 \\
\hline $\mathrm{Na}_{2} \mathrm{SO}_{4}$ & -0.380 & 1.27 & 0.8 & 3.6 & 3.6 \\
\hline $\mathrm{K}_{2} \mathrm{SO}_{4}$ & -0.200 & 1.07 & 0.55 & 3.1 & 3.1 \\
\hline $\mathrm{Rb}_{2} \mathrm{SO}_{4}$ & -0.341 & 1.33 & 0.5 & $3.7_{5}$ & 3.8 \\
\hline $\mathrm{Cs}_{2} \mathrm{SO}_{4}$ & -0.260 & 1.33 & 0.5 & 3.8 & 3.8 \\
\hline
\end{tabular}

${ }^{b}$ See below Table 1 .

is considerably lower than the conductivity of $\mathrm{K}^{+}$ (5.54/7.35), so $\mathrm{F}^{-}$is probably more hydrated than $\mathrm{K}^{+}$due to the ability of this ion to form hydrogen bonds. It does not seem possible to decompose the contact distances into efficient ionic radii in any unique way consistent with both the data for $a$ (DHEV I) and conductivity data, not even for the ions with low or moderate electron numbers. With a square-well attractive potential, it may well be possible to find consistent hard sphere radii and attractive potentials, but this will not be attempted here.

(5) The value of $a$ (DHEV I) for $\mathrm{LiNO}_{3}$ is identical to the $a$ (DHEV I) for $\mathrm{LiCl}$ (3.8). This is well in accordance with the almost identical conductivities for $\mathrm{Cl}^{-}$and $\mathrm{NO}_{3}^{-}$. However, the contact distances for $\mathrm{NaNO}_{3}, \mathrm{KNO}_{3}, \mathrm{RbNO}_{3}$ and $\mathrm{CsNO}_{3}$ are less than for the corresponding chlorides, and the deviations become worse through the series. This is well in accordance with earlier considerations of Kelbg (see Ref. 10, p. 424, Fig. 121), who found it necessary to intro- duce a square-well potential for the alkali nitrates. The value of $\tau$ [see eqn. (10)] correlated well with the number of electrons in the cation. For $\mathrm{LiNO}_{3}, \tau$ was only 0.04 whereas $\tau=0.30$ for $\mathrm{KNO}_{3}$.

(6) The $a$ (DHEV I) for $\mathrm{Mg}\left(\mathrm{NO}_{3}\right)_{2}, \mathrm{Co}\left(\mathrm{NO}_{3}\right)_{2}$ and $\mathrm{Ni}\left(\mathrm{NO}_{3}\right)_{2}$ is almost identical to the contact distances for the corresponding chlorides, whereas negative deviations are found for $\mathrm{Ca}\left(\mathrm{NO}_{3}\right)_{2}$ and $\mathrm{Sr}\left(\mathrm{NO}_{3}\right)_{2}$. Again, this may be correlated with the number of electrons in the cation.

(7) The contact distance for $\mathrm{NH}_{4} \mathrm{Cl}$ is identical to the contact distance for $\mathrm{KCl}(3.15)$ in accordance with conductivity data. However, $\&$ (DHEV I, $\left.\mathrm{NH}_{4} \mathrm{NO}_{3}\right)<\AA\left(\mathrm{DHEV} \mathrm{I}, \mathrm{NH}_{4} \mathrm{Cl}\right)$ although the conductivities are almost identical.

(8) The contact distances for $\mathrm{LiOH}, \mathrm{NaOH}$, $\mathrm{KOH}$ and $\mathrm{CsOH}$ follow roughly the sum of crystal radii (assuming a radius for $\mathrm{OH}^{-}$equal to $\mathrm{O}^{-}$). A plausible explanation is, that when $\mathrm{OH}^{-}$ approaches for example a hydrated $\mathrm{Li}^{+}$-ion, then it exchanges a proton with the bound water

Acta Chem. Scand. A 38 (1984) No. 10 
Table 3. Comparison between sum of crystal ionic radii (from Ref. 34) and contact distances from DHEV theory.

\begin{tabular}{|c|c|c|c|c|}
\hline $\begin{array}{l}\text { Elec- } \\
\text { trolyte }\end{array}$ & $\begin{array}{l}\text { Number of } \\
\text { electrons } \\
\text { cation }\end{array}$ & anion & $\begin{array}{l}R_{12}(\text { crystal }) \\
(0.1 \mathrm{~nm})\end{array}$ & $\begin{array}{l}a(D H E V ~ I) \\
(0.1 \mathrm{~nm})\end{array}$ \\
\hline $\begin{array}{l}\mathrm{LiCl} \\
\mathrm{LiBr} \\
\mathrm{LiI} \\
\mathrm{NaF} \\
\mathrm{NaCl} \\
\mathrm{NaBr} \\
\mathrm{NaI} \\
\mathrm{KF} \\
\mathrm{KCl} \\
\mathrm{KBr} \\
\mathrm{KI} \\
\mathrm{RbCl} \\
\mathrm{RbBr} \\
\mathrm{RbI} \\
\mathrm{CsCl} \\
\mathrm{CsBr} \\
\mathrm{CsI} \\
\mathrm{LiOH} \\
\mathrm{NaOH} \\
\mathrm{KOH} \mathrm{CHOH} \\
\mathrm{CsOH} \\
\mathrm{MgCl}_{2} \\
\mathrm{MgBr}_{2} \\
\mathrm{CaCl}_{2} \\
\mathrm{CaBr}_{2} \\
\mathrm{SrCl}_{2} \\
\mathrm{SrBr}_{2} \\
\mathrm{BaCl}_{2} \\
\mathrm{BaBr}_{2} \\
\mathrm{MnCl}_{2} \\
\mathrm{FeCl}_{2} \\
\mathrm{CoCl}_{2} \\
\mathrm{NiCl}_{2} \\
\mathrm{CuCl}_{2}\end{array}$ & $\begin{array}{r}2 \\
2 \\
2 \\
10 \\
10 \\
10 \\
10 \\
18 \\
18 \\
18 \\
18 \\
36 \\
36 \\
36 \\
54 \\
54 \\
54 \\
2 \\
10 \\
18 \\
54 \\
10 \\
10 \\
18 \\
18 \\
36 \\
36 \\
54 \\
54 \\
23 \\
24 \\
25 \\
26 \\
27\end{array}$ & $\begin{array}{r}18 \\
36 \\
54 \\
10 \\
18 \\
36 \\
54 \\
10 \\
18 \\
36 \\
54 \\
18 \\
36 \\
54 \\
18 \\
36 \\
54 \\
8 \\
8 \\
8 \\
8 \\
18 \\
36 \\
18 \\
36 \\
18 \\
36 \\
18 \\
36 \\
18 \\
18 \\
18 \\
18 \\
18\end{array}$ & $\begin{array}{l}2.5 \\
2.6 \\
2.9 \\
2.3 \\
2.8 \\
2.9 \\
3.2 \\
2.7 \\
3.1 \\
3.3 \\
3.5 \\
3.3 \\
3.4 \\
3.7 \\
3.5 \\
3.6 \\
3.9 \\
2.4^{\mathrm{a}} \\
2.7 \\
3.1 \\
3.4 \\
2.5 \\
2.6 \\
2.8 \\
2.9_{5} \\
2.9 \\
3.1 \\
3.1_{5} \\
3.3 \\
2.6 \\
2.5_{5} \\
2.5 \\
2.5 \\
2.5\end{array}$ & $\begin{array}{l}3.5 \\
3.65 \\
3.9 \\
3.0 \\
3.3 \\
3.4 \\
3.5 \\
3.25 \\
3.15 \\
3.2 \\
3.3 \\
3.0 \\
3.0 \\
2.9 \\
2.8 \\
2.8 \\
2.7 \\
1.9 \\
3.0 \\
3.3 \\
4.0 \\
4.7 \\
4.9 \\
4.6 \\
4.8 \\
4.6 \\
4.8 \\
4.55 \\
4.6 \\
4.6 \\
4.6 \\
4.6 \\
4.6 \\
4.5 \\
\end{array}$ \\
\hline
\end{tabular}

${ }^{a}$ Under the assumption that the crystal ionic radius of $\mathrm{OH}^{-}$is identical to the radius of $\mathrm{O}^{-}$.

molecules. Therefore, the hydration shell will not be a "barrier" for $\mathrm{OH}^{-}$.

(9) The divalent cations $\mathrm{Mg}^{2+}, \mathrm{Ca}^{2+}, \mathrm{Sr}^{2+}$, $\mathrm{Ba}^{2+}, \mathrm{Mn}^{2+}, \mathrm{Fe}^{2+}, \mathrm{Co}^{2+}, \mathrm{Ni}^{2+}$, and $\mathrm{Cu}^{2+}$ all seem to have the same size (with hydration), namely $R_{12}=0.46 \mathrm{~nm}$, when opposed to $\mathrm{Cl}^{-}$, although the crystal ionic radii vary somewhat (from 0.25 to $0.32 \mathrm{~nm}$ ). Their conductivities are also quite similar (below $20 \%$ deviations).

(10) The contact distances found for the Rband Cs-halogenides are less than the sum of crystal ionic radii. This points to significant attractive forces. Those deviations increase from $\mathrm{Rb}^{+}$to $\mathrm{Cs}^{+}$and from $\mathrm{Cl}^{-}$to $\mathrm{I}^{-}$, so they correlate with number of electrons in cation and anion. In conclusion the attractive forces between ions become of significance from an (18/18) electron distribution $(\mathrm{KCl})$. Attractive forces are due to ion-induced dipole forces, and the polarisability of the electrons in the higher shells increases substantially. To a minor extent, attractive forces may also arise from London- van der Waal's

Acta Chem. Scand. A 38 (1984) No. 10 
forces, which correlate directly with the number of electrons.

It seems that the simple hard sphere additivity is somewhat destroyed by the attractive forces. It should be noticed, however, that attractive forces might not be the only scapegoat. In the DHEV I theory, we have taken all excluded volumes into account, but the DH-denominator summarizes only in a crude way all the other terms in for example eqn. (29). Perhaps, the most serious omission is the linear term in the Bjerrumparameter dependent on the square of the difference between the ionic radii. The more detailed comparison between experimental data and the Schmitz theory or MSA-theory will be postponed to a future paper, which will deal also with activity coefficients in mixtures of electrolytes. Here, we shall be content with the statement, that the DHEV I theory yields contact distances with a certain, although not perfect, consistency.

\section{IONIC RADII FROM ILEV I AND ILEV II}

Already in 1918, Ghosch ${ }^{35}$ proposed a model for strong electrolytes in solution based on the assumption that the ions in the solution in time-average will be placed as the ions in a crystal lattice "pumped up" with solvent. The theory leads to a dependence of $\ln y_{ \pm}$on the cube root of the concentration instead of the square root dependence of the DH-theory. Therefore, the Ghosch theory is incorrect as a limiting law. On the other hand, it has often been pointed out, that the cube root law is followed over a much broader concentration range than the $\mathrm{DH}$ limiting law. However, false extrapolations to zero concentrations is the inevitable consequence. (This leads to somewhat wrong $\mathrm{pH}$ values in the buffers standardized by S.P.L. Sørensen). The lattice theory was proposed independently and simultaneously by Bjerrum ${ }^{36}$ and later generalized by Ghosch. ${ }^{37}$ In 1939 , Prigogine ${ }^{38}$ investigated the transition from the random ionic cloud structure at low concentrations to the more lattice-like structure at higher concentrations introducing a "degree of order". Various modifications of the lattice theory have been proposed by Frank and Thompson, ${ }^{39}$ Desnoyers and Conway, ${ }^{40}$, Glueckauf ${ }^{41}$ and Bahe. ${ }^{42}$ Recently, these approaches have been reviewed by Pytko- wicz and Johnson, ${ }^{43}$ who propose still other modifications.

In the present paper, we shall just show that a lattice theory supplemented with excluded volume terms fits well with the experiments from moderate dilution up to $3-4 \mathrm{~mol} / \mathrm{dm}^{3}$ for a number of 1:1 and 2:1 electrolytes (halogenides), and the radii (contact distances) obtained from the theory are not far from the DHEV-radii.

The electrostatic energy per mol of a crystal lattice is given by: ${ }^{44}$

$E_{\mathrm{m}}^{\mathrm{el}}=-\left|z_{1} \cdot z_{2}\right| \cdot e_{\mathrm{o}}^{2} \cdot M \cdot N_{\mathrm{o}} /\left(4 \pi \cdot \varepsilon \cdot r_{\mathrm{o}}\right)$

$M$ is the Madelung constant for the given type of crystal lattice, and $r_{\mathrm{o}}$ is the shortest distance (in meters) between two oppositely charged ions in the lattice. The idea is to use a similar expression for solutions with $r_{\mathrm{o}}$ being the average distance between neighbouring ions of opposite charge. For 1:1 electrolytes we assume a $\mathrm{NaCl}$ lattice (Fig. 2) although the Cs-halogenides are known to crystallize in a CsCl-lattice. The Madelung constants are only slightly different for the two types of lattices (1.748 and 1.763). For the 2:1 electrolytes we assume a Fluorite $\left(\mathrm{CaF}_{2}\right)$ type of lattice (Fig. 3). The Madelung constant for this lattice is 2.519 .

If we consider $n \mathrm{~mol}$ of electrolyte, we have $v \cdot n \cdot N_{\mathrm{o}}$ ions, and if we have $\mathrm{x}$ ions in the unit cell, the volume occupied by the $n \mathrm{~mol}$ is $1000 \cdot v \cdot n \cdot N_{\mathrm{o}} \cdot l^{3} / x \mathrm{dm}^{3}$, with $l$ being the edge length in meters of the (cubic) unit cell. Thus, we have

$c_{\mathrm{s}}=x /\left(1000 \cdot v \cdot N_{\mathrm{o}} \cdot l^{3}\right)$

For $1: 1$ electrolytes we have $v=2$ and also $x=1$ and $l=r_{0}=$ distance between nearest neighbours

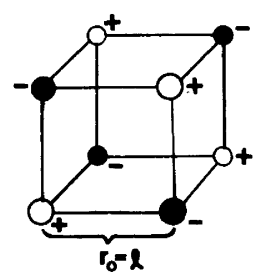

Fig. 2. Geometry used for calculation of electrostatic free energy in solutions of $1: 1$ electrolytes: The $\mathrm{NaCl}$ lattice. Madelung constant 1.748. 


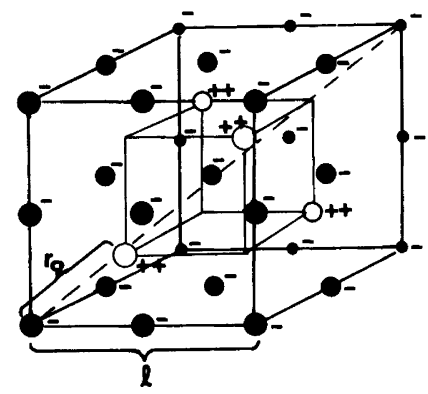

Fig. 3. Geometry of $\mathrm{CaF}_{2}$ lattice used for solutions of 2:1 electrolytes. Madelung constant 2.519 .

of opposite charge (see Fig. 2). For 2:1 electrolytes we have $v=3, x=12$ and $l=4 \cdot r_{\mathrm{o}} / \sqrt{3}$ (see Fig. 3 and notice that the diagonal in the unit cell is equal to $4 r_{\mathrm{o}}$ ). Using those relations in connection with eqns. (78) and (79) we obtain:

$E_{\mathrm{m}}^{\mathrm{el}}=\left\{\begin{array}{l}-\frac{e_{\mathrm{o}}^{2} \cdot 10 \cdot 2^{\frac{1}{3}} \cdot N_{\mathrm{o}}^{\frac{4}{3}}}{4 \pi \cdot \varepsilon} \cdot M(1: 1) \cdot \sqrt[3]{c_{\mathrm{s}}} \\ -\frac{2 e_{\mathrm{o}}^{2} \cdot 10 \cdot 4^{\frac{2}{3}} \cdot N_{\mathrm{o}}^{\frac{4}{3}}}{4 \pi \cdot \varepsilon \cdot 3^{\frac{1}{2}}} \cdot M(2: 1) \cdot \sqrt[3]{c_{\mathrm{s}}}\end{array}\right.$

For the electrostatic contribution to the mean ionic activity we have

$E_{\mathrm{m}}^{\mathrm{el}}=v \cdot R T \cdot \ln y_{ \pm}^{\mathrm{el}}$

since $E_{m}^{e l}$ can be interpreted as an excess molar free energy. Using eqns. (80) and (81) and inserting values at $25^{\circ} \mathrm{C}$ and the mentioned Madelung constants we finally obtain $\ln y_{ \pm}^{\mathrm{el}}=-A_{\mathrm{L}} \cdot \sqrt[3]{\mathrm{c}_{\mathrm{s}}}$

with the lattice constants given by:

$A_{\mathrm{L}}=\left\{\begin{array}{l}0.664\left(1: 1,25^{\circ} \mathrm{C}\right) \\ 1.472\left(2: 1,25^{\circ} \mathrm{C}\right)\end{array}\right.$

It should be remarked that the values in eqn. (83) correspond to $\varepsilon=6.954 \cdot 10^{-10} \mathrm{C} \cdot \mathrm{V}^{-1} \cdot \mathrm{m}^{-1}$ for pure water at $25^{\circ} \mathrm{C}$. However, in principle eqn. (80) is valid at any absolute permittivity (with $\varepsilon$ assumed uniform), so it is relatively easy to correct for the decrease in dielectric constant with the increase in salt concentration in the lattice model.

The Ionic Lattice/Excluded Volume (ILEV) model is expressed through the following equation:

$\ln y_{ \pm}=k_{\mathrm{L}}-A_{\mathrm{L}} \cdot \sqrt[3]{c_{\mathrm{s}}}+B_{\mathrm{L}} \cdot c_{\mathrm{s}}$

The empirical constant $k_{L}$ corrects for the deviation from the DH limiting law at low concentrations. In ILEV I, all excluded volumes are incorporated in the $\mathrm{B}_{\mathrm{L}}$-coefficient, whereas the Brønsted principle is used in ILEV II. The semiempirical formula (84) fits the empirical data very well from moderately dilute to quite concentrated solutions (below 0.1 to $2-3 \mathrm{~mol} / \mathrm{dm}^{3}$ or even more), see the plots for $\mathrm{Li}$ - and $\mathrm{Ca}$ halogenides given in Figs. 4 and 5.

An equation similar to (84), but without $B_{\mathrm{L}}$, was derived by Frank and Thomson. ${ }^{39}$ The

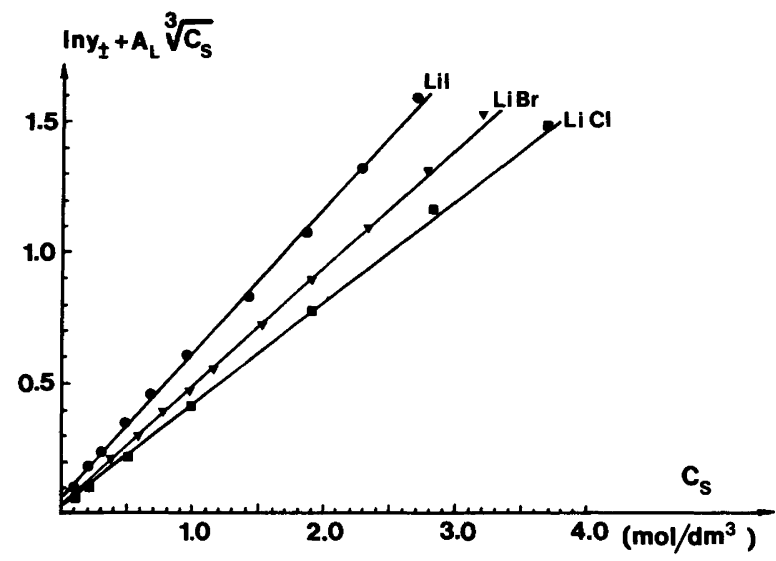

Fig. 4. ILEV plot for Li-halogenides in water at $25^{\circ} \mathrm{C}$.

Acta Chem. Scand. A 38 (1984) No. 10 


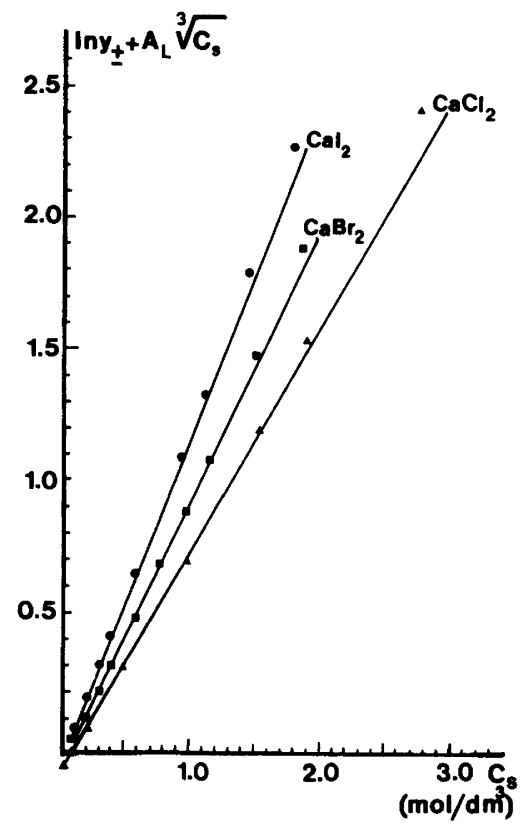

Fig. 5. ILEV plot for Ca-halogenides in water at $25^{\circ} \mathrm{C}$.

equation reached by Bahe is eqn. (84) without $k_{\mathrm{L}}$. Bahe ${ }^{42}$ considered the interaction between the gradient in dielectric constant near an ion immersed in water and the electric field around another ion. Those interactions lead to repulsive forces between the ions according to Bahe. Bahe's model is criticized by Pytkowicz and
Johnson, ${ }^{43}$ which authors instead propose an equation similar to (84) but on a molality basis (and with $\ln \gamma_{ \pm}$). They introduce other dielectric explanations for the $\mathrm{B}_{\mathrm{L}}$-coefficients. It seems not to have occurred to any authors that the Bcoefficient might be simply an excluded volume term. Furthermore, Pytkowicz and Johnson add to the general confusion in deviating in their $A_{L}$-values from the ones given here, thus deviating from the original method of Ghosch and Bjerrum. In our opinion their $A_{\mathrm{L}}$-values, based on a superfluous differentiation of $E_{\mathrm{m}}^{\text {el }}$ with respect to concentration (Ref. 43 p. 234), are false. For 1:1 electrolytes they obtain $A_{\mathrm{L}}=0.887$ instead of 0.664 . Attempts to plot $\ln y_{ \pm}-0.887$. $\sqrt[3]{c_{\mathrm{s}}}$ against $c_{\mathrm{s}}$ lead to great deviations from linearity, see Fig. 6.

The same data for $\ln \gamma_{ \pm}$as used in Refs. 24 and 25 were used, but eqn. (67) was used for the transformation to $\ln y_{ \pm}$instead of the slightly incorrect formula used in Refs. 24 and 25 . The values of $k_{\mathrm{L}}$ and $B_{\mathrm{L}}$ for a number of electrolytes are listed in Table 4 together with the concentration range, where eqn. (84) can be used with a precision corresponding to \pm 0.02 (mostly below \pm 0.01 ) on $\ln y_{ \pm}$. The calculated contact distances for ILEV I and ILEV II are also listed. In contrast to the DHEV 'contact distances, ILEV I and ILEV II distances differ considerably. ILEV I contact distances come closest to the DHEV distances, although they are somewhat larger. For the light cations (e.g. $\mathrm{H}^{+}, \mathrm{Li}^{+}, \mathrm{Mg}^{2+}$ ), the values of $a$ (ILEV I) are

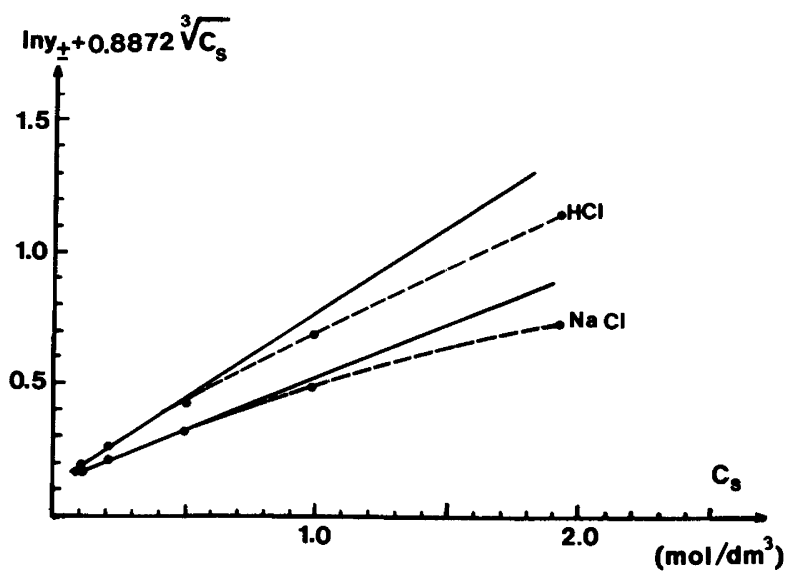

Fig. 6. Deviations of ILEV plots from linearity using $A_{\mathrm{L}}=0.887$ (Pytkowicz and Johnson) instead of 0.664 (this work). 
Table 4. ILEV parameters and contact distances for various electrolytes in water at $25^{\circ} \mathrm{C}$.

\begin{tabular}{|c|c|c|c|c|c|}
\hline $\begin{array}{l}\text { Elec- } \\
\text { trolyte }\end{array}$ & $k_{\mathrm{L}}$ & $\begin{array}{l}B_{\mathrm{L}} \\
\mathrm{dm}^{3} / \mathrm{mol}\end{array}$ & $\begin{array}{l}\text { Certified } \\
\text { conc. range } \\
\text { mol } / \mathrm{dm}^{3}\end{array}$ & $\begin{array}{l}a(\text { ILEV I })^{\mathrm{b}} \\
\delta=1 \\
0.1 \mathrm{~nm}\end{array}$ & $\begin{array}{l}\stackrel{\circ}{a}(\text { ILEV II }) \\
\delta=0 \\
0.1 \mathrm{~nm}\end{array}$ \\
\hline $\mathrm{HCl}$ & 0.039 & 0.437 & $0.01-3.7$ & 4.2 & 5.6 \\
\hline $\mathrm{HBr}$ & 0.044 & 0.520 & $0.01-2.5$ & 4.5 & 5.9 \\
\hline HI & 0.056 & 0.620 & $0.1-2.3$ & 4.7 & 6.3 \\
\hline $\mathrm{LiCl}$ & 0.025 & 0.388 & $0.01-3.7$ & 4.0 & 5.4 \\
\hline $\mathrm{LiBr}$ & 0.038 & 0.440 & $0.1-2.8$ & 4.2 & 5.6 \\
\hline LiI & 0.070 & 0.542 & $0.1-2.5$ & 4.5 & 6.0 \\
\hline $\mathrm{NaCl}$ & 0.040 & 0.220 & $0.01-3.7$ & 3.4 & 4.4 \\
\hline $\mathrm{NaBr}$ & 0.034 & 0.283 & $0.2-3.6$ & 3.8 & 4.8 \\
\hline $\mathrm{NaI}$ & 0.043 & 0.355 & $0.1-3.1$ & 4.0 & 5.2 \\
\hline $\mathrm{KCl}$ & 0.034 & 0.155 & $0.05-3.5$ & 3.1 & 4.0 \\
\hline $\mathrm{KBr}$ & 0.035 & 0.177 & $0.05-3.5$ & 3.3 & 4.1 \\
\hline $\mathrm{KI}$ & 0.043 & 0.223 & $0.1-3.7$ & 3.5 & 4.5 \\
\hline $\mathrm{RbCl}$ & 0.013 & 0.144 & $0.2-4.2$ & 3.1 & 3.9 \\
\hline $\mathrm{RbBr}$ & 0.014 & 0.140 & $0.3-4.1$ & 3.0 & 3.8 \\
\hline RbI & -0.007 & 0.163 & $0.2-3.9$ & 3.2 & 4.0 \\
\hline $\mathrm{MgCl}_{2}$ & -0.052 & 1.004 & $0.1-1.5$ & 5.3 & 6.7 \\
\hline $\mathrm{MgBr}_{2}$ & -0.052 & 1.260 & $0.1-1.5$ & 5.8 & 7.2 \\
\hline $\mathrm{MgI}_{2}$ & -0.018 & 1.452 & $0.1-1.3$ & 6.0 & 7.6 \\
\hline $\mathrm{CaCl}_{2}$ & -0.062 & 0.892 & $0.1-2.0$ & 5.2 & 6.4 \\
\hline $\mathrm{CaBr}_{2}$ & -0.055 & 1.075 & $0.1-1.7$ & 5.5 & 6.8 \\
\hline $\mathrm{CaI}_{2}$ & -0.033 & 1.293 & $0.1-1.7$ & 5.9 & 7.3 \\
\hline $\mathrm{SrCl}_{2}$ & -0.057 & 0.808 & $0.1-2.2$ & 5.0 & 6.2 \\
\hline $\mathrm{SrBr}_{2}$ & -0.049 & 0.963 & $0.1-1.8$ & 5.3 & 6.6 \\
\hline $\mathrm{SrI}_{2}$ & -0.030 & 1.212 & $0.1-1.5$ & 5.7 & 7.1 \\
\hline $\mathrm{BaCl}_{2}$ & -0.052 & 0.648 & $0.1-1.7$ & 4.7 & 5.8 \\
\hline $\mathrm{BaBr}_{2}$ & -0.054 & 0.848 & $0.1-1.8$ & 5.1 & 6.3 \\
\hline $\mathrm{BaI}_{2}$ & -0.049 & 1.189 & $0.1-1.7$ & 5.7 & 7.1 \\
\hline
\end{tabular}

${ }^{a} \pm 0.02$ (mostly \pm 0.01 ) on $\ln y_{ \pm} \cdot{ }^{b}$ Values of $r$ as in Tables 1 and 2.

about 0.5 units (i.e. $0.05 \mathrm{~nm}$ ) larger than $\stackrel{\circ}{a}$ (DHEV I). For the more heavy cations the correspondence is quite close. This means, that the problems in comparing with $R_{12}$ (cryst) are not resolved by the ILEV model. On the other hand, it is comforting to obtain almost identical "effective" contact distances from two widely different models covering widely different concentration ranges.

If we use $B$ (DHEV I) instead of $B_{L}$ in eqn. (84), one may calculate an "effective" value of $A_{\mathrm{L}}$ as a function of concentration. From the ratio $A_{\mathrm{L}}$ (infinite dilution) $/ \mathrm{A}_{\mathrm{L}}$ (eff) it is possible to estimate the ratio between the "effective" dielectric constant and the dielectric constant for the pure solvent. This has been done for $\mathrm{NaCl}$ in water at $25^{\circ} \mathrm{C}$.

The calculated effective dielectric constant fluctuates wildly at higher concentrations, and it seems rather greater than the dielectric constant of pure water than less. Therefore, it is not possible to point towards dielectric constant decrease with increasing concentration as the reason for the deviation between the values of B(ILEV I) and the values of B(DHEV I).

On the other hand, the decrease of the dielectric constant has repeatedly been quoted by electrochemists as a factor influencing electrolyte activities, since Hückel connected the dielectric constant decrease with the " $B$-terms" (proportional to $c_{s}$ ) in the expression for $\ln y_{ \pm}$(see Ref. $45 \S 6$ ). For $\mathrm{NaCl}$ solutions in water, the macroscopic dielectric constant depends approximately linearly with concentration: ${ }^{46}$

$\varepsilon($ solution $) / \varepsilon($ water $) \cong 1-0.1185 \cdot c_{\mathrm{s}}$ 


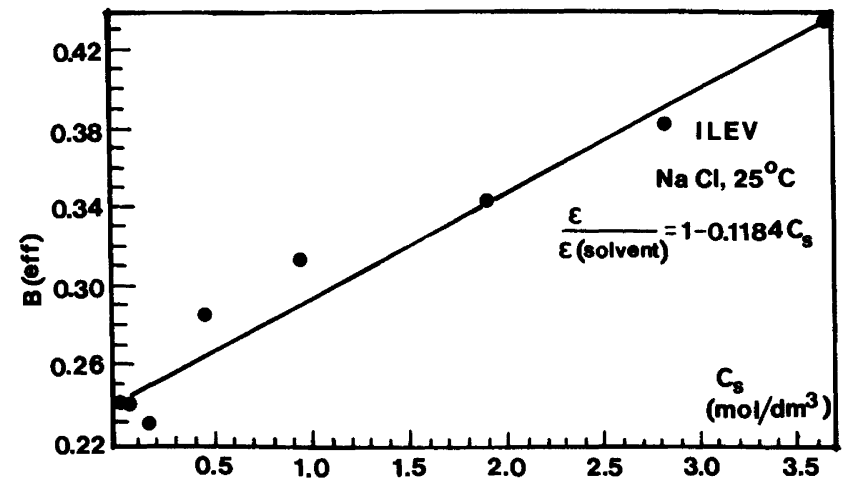

Fig. 7. Effective values of excluded volume taking into account dielectric constant decrease with increasing salt concentration. $\mathrm{NaCl}$ in water at $25^{\circ} \mathrm{C}$.

From eqn. (85), an $A_{\mathrm{L}}($ eff) may be calculated, and from eqn. (84) and the experimental values of $\ln y_{ \pm}$we may calculate "effective" values of $B_{\mathrm{L}}$ as a function of $c_{\mathrm{s}}$. This approach yields much more consistent results, see Fig. 7. However, we are now left with the problem of explaining the great increase in the "excluded volume" with concentration.

One plausible explanation would be the following: From the theory of Schmitz we know, that for small plasma parameters (high dilution), the "effective" excluded volumes may well be larger than the hard sphere excluded volumes, when the Bjerrum parameters are not small compared to unity, see eqns. (19) and (22). Although the validity of the Schmitz theory is not warranted at higher concentrations, the theory may well describe the tendency with respect to the Bjerrum parameter in a correct manner. Now, at higher salt concentrations there will exist a competition between $\mathrm{Cl}^{-}$and the hydrate water around $\mathrm{Na}{ }^{+}$ Some bound water molecules may exchange with $\mathrm{Cl}^{-}$. We have therefore a smaller contact distance for some of the ion pairs. This distance is approximately $R_{12}$ (cryst) $=0.28 \mathrm{~nm}$. According to the original theory of Bjerrum (see Ref. $10 \mathrm{p}$. 302), two ions are regarded as "bound" for contact distances smaller than around $0.35 \mathrm{~nm}$, and then we cannot assume small Bjerrum parameter. We therefore have the paradox, that the "effective" excluded volumes may increase with concentration, because the hard sphere excluded volumes decrease with concentration! However, in view of all the uncertainties given, we cannot push the calculations further at this point. We shall just finish this section by pointing out, that in the DHEV theory the compensating effect of larger Bjerrum parameters is to a certain extent taken care of by the DH-denominator, which is equivalent to the quadratic term in the Bjerrum parameter. This denominator is lacking in the ILEV theory. The success of the latter theory to describe data over an extended range of concentrations may well be due to the simultaneous neglect of Bjerrum effects and decrease of dielectric constant.

Very recently, Ruff and co-workers ${ }^{49-52}$ have proposed a more refined lattice theory than that of Bahe ${ }^{42}$ They use Booth's theory ${ }^{53,54}$ for the dependence of the "microscopic" dielectric constant on the electric field strength in calculating the average permittivity in the lattice. However, the ions are always taken as point charges, and the dielectric lowering due to the presence of "ionic volumes" with lower dielectric constant than water is not taken into account in the averaging. The authors claim to fit activity coefficients without any adjustable parameters. It should be mentioned, however, that Booth's equation is not generally agreed upon, and it seems unrealistic to omit excluded volumes. The fit to activity data for 1:1, 2:1 and 1:2 electrolytes is actually very bad, see Ref. 49, Figs. 12 and 13 . The theory has some merits, however, in giving an explanation for Harned's rule for mixed electrolytes, ${ }^{50}$ in discussing the lattice/DebyeHückel transition at low ionic strengths ${ }^{51}$ and in yielding realistic, oscillating charge densities around ions. ${ }^{52}$ The theory also points out the importance of "solvation forces" between ions as 
stabilizing forces opposing ion pair formation and crystallization. This seems to be an important step towards a more realistic description, in which water is not only treated as a "dielectric continuum" without structure! A detailed account of the theory of Ruff is outside the scope of the present paper, however.

\section{ACTIVITIES FROM ASPEV FORMULAE AT VARYING TEMPERATURES}

In two previous papers ${ }^{24,25}$ it was noticed that $\ln y_{ \pm}$for alkali- and earth alkali halogenides exhibited a parabolic dependence on $\sqrt{I}$ at least from the activity coefficient minimum to somewhat above the pseudo-ideal point, where higher virial coefficients come in (or where the salt precipitates). The $B \cdot I$ term was linked to the excluded volume between anion and cation assuming the Brønsted principle to be valid.

However, the terms describing the transition towards the parabola for the lower salt concentrations were somewhat unfortunately selected, since there was no smooth transition to the DHLL. In the meantime this has been remedied, and new fittings on the experimental data have been made. We shall maintain the name ASPEV (=Adjusted Screened Potential Excluded Volume) since the basic idea is, that the effective potentials at the position of an ion is screened by the presence of counterions, so that the electrostatic interaction decreases with increasing concentration (This is brought out more quantitatively in the MSA theory, where the shielding length $\Gamma^{-1}$ is larger than the DH shielding length $\left.\boldsymbol{\kappa}^{-1}\right)$. The word "adjusted" is taken to mean just the empirical fitting to experimental data, which is made necessary by the confusing amount of possible effects at higher salt concentrations: Dielectric constant lowering and gradients near ions, various attractive potentials between ions, hydration layer penetration of counterions and corresponding increase in Bjerrum parameters, more realistic repulsion potentials than the hard sphere potential and the effect of the structure of the water itself.

The new fittings have been performed also for temperatures different from $25^{\circ} \mathrm{C}$ for some few electrolytes. The modified ASPEV formulae have recently been published, but only in
Danish. ${ }^{47}$ For the mean molar activity coefficient we write:

$$
\begin{aligned}
& \ln y_{ \pm}=-A^{*} \sqrt{I}+B^{*} I \\
& A^{*}=A_{\min }^{*}+\left\{\left|z_{1} z_{2}\right| A_{\mathrm{DH}}-A_{\min }^{*}\right\} \cdot \exp (-\alpha \sqrt{I})
\end{aligned}
$$

$A_{\mathrm{DH}}=\frac{6037}{T^{\frac{3}{2}}\left(\varepsilon_{\mathrm{r}} / 78.54\right)^{\frac{3}{2}}}\left(\mathrm{in} \mathrm{dm}^{3 / 2} \mathrm{~mol}^{-\frac{1}{2}}\right)$

Temperature dependence of relative permittivity of pure water:

$$
\begin{aligned}
& \frac{\varepsilon_{\mathrm{r}}}{78.54}=1-4.579 \cdot 10^{-3}(t-25)+11.9 \cdot 10^{-6}(t-25)^{2} \\
& +28 \cdot 10^{-9}(t-25)^{3} \quad\left(t \text { in }{ }^{\circ} \mathrm{C}\right)
\end{aligned}
$$

Terms connected with excluded volume:

$B^{*}=n \cdot \frac{4 \pi N_{\mathrm{o}}}{3} \cdot R_{12}^{3} \cdot 10^{-27}\left(\mathrm{dm}^{3} / \mathrm{mol}\right) \quad\left(\mathrm{R}_{12}\right.$ in $\left.\AA\right)$

$n= \begin{cases}1 & (1: 1) \\ 4 / 9 & (2: 1)\end{cases}$

$B^{*}=n \cdot 2.52 \cdot 10^{-3} \cdot R_{12}^{3}$

( $B^{*}$ in $\mathrm{dm}^{3} \mathrm{~mol}^{-1}, R_{12}$ in $\AA$ )

$\left.\begin{array}{l}A^{*}{ }_{\min }=0.73-8 \cdot 10^{-4} \cdot t\left(t \text { in }{ }^{\circ} \mathrm{C}\right) \\ \alpha=0.64 \cdot\left(1-4 \cdot 10^{-3} t\right) \cdot R_{12}\left(R_{12} \text { in } \AA\right)\end{array}\right\}(1: 1)$

$A^{*}{ }_{\min }=1.28 ; \alpha=0.40 \cdot R_{12}$

( $\alpha$ in $\mathrm{dm}^{\frac{3}{2}} \mathrm{~mol}^{-\frac{1}{2}}$, only $25^{\circ} \mathrm{C}$ )

The ASPEV contact distances are listed in Table 5 for all combinations of $(\mathrm{H}, \mathrm{Li}, \mathrm{Na}, \mathrm{K}$, $\mathrm{Rb}, \mathrm{Cs}, \mathrm{Mg}, \mathrm{Ca}, \mathrm{Sr}, \mathrm{Ba})$ with $(\mathrm{Cl}, \mathrm{Br}, \mathrm{I})$ at $25^{\circ} \mathrm{C}$. The maximum errors are somewhat larger than for the ILEV expression, but the ASPEV formulae contain only one adjustable parameter $\left(R_{12}\right)$, whereas ILEV has two $\left(k_{\mathrm{L}}\right.$ and $\left.B_{\mathrm{L}}\right)$. Furthermore, ASPEV passes smoothly over into DHLL in contrast to ILEV [replace the exponential in eqn. (87) by unity and omit $B^{*}$ in eqn. (86)]. It can even be shown, that the ASPEV formula is equivalent to the DHEV II (Brønsted assumption is used for simplicity) at moderate dilution. The exponential in eqn. (87) is replaced by $1-\alpha \sqrt{I}$ and the $\sqrt{I}$ and $I$ terms are compared with the corresponding terms in the expansion of the DHEV II expression. We obtain: 
Table 5. Contact distances from the ASPEV theory in water at $25^{\circ} \mathrm{C}$ for some $1: 1$ and $2: 1$ electrolytes $\left(R_{12}\right.$ in units of $\left.0.1 \mathrm{~nm}\right)$.

\begin{tabular}{lllll}
\hline & $\mathrm{Cl}^{-}$ & $\mathrm{Br}^{-}$ & $\mathrm{I}^{-}$ & Error on ln $y_{ \pm}$ \\
\hline $\mathrm{H}^{+}$ & 5.98 & 6.28 & 6.62 & less than \pm 0.02 for $1: 1$ \\
$\mathrm{Li}^{+}$ & 5.73 & 6.00 & 6.41 & electrolytes up to \\
$\mathrm{Na}^{+}$ & 5.09 & 5.33 & 5.68 & 2 mol $/ \mathrm{dm}^{3}$. (For salts which \\
$\mathrm{K}^{+}$ & 4.72 & 4.84 & 5.13 & do not precipitate, the \\
$\mathrm{Rb}^{+}$ & 4.55 & 4.55 & 4.57 & formulae may be used at \\
$\mathrm{Cs}^{+}$ & 4.20 & 4.20 & 4.20 & $\begin{array}{l}\text { greater conc. with somewhat } \\
\text { greater uncertainty.) }\end{array}$ \\
& & & & \\
$\mathrm{Mg}^{2+}$ & 7.94 & 8.37 & 8.70 & less than \pm 0.05 up to \\
$\mathrm{Ca}^{2+}$ & 7.70 & 8.05 & 8.40 & $1.33 \mathrm{~mol} / \mathrm{dm}^{3}\left(I=4 \mathrm{~mol} / \mathrm{dm}^{3}\right)$ \\
$\mathrm{Sr}^{2+}$ & 7.60 & 7.90 & 8.30 & for $2: 1$ electrolytes. \\
$\mathrm{Ba}^{2+}$ & 7.40 & 7.70 & 8.24 & $\left( \pm 0.2\right.$ up to $\left.5 \mathrm{~mol} / \mathrm{dm}^{3}\right)$ \\
\hline
\end{tabular}

The values of $R_{12}$ correspond to best fits to the same experimental data as quoted in Refs. 24 and 25 . Transformation from $\gamma_{ \pm}$to $y_{ \pm}$by means of eqn. (67) and formulae for $c_{\mathrm{s}} / m_{\mathrm{s}}$ in Ref. 26, App. A.

$B^{*}=B(\mathrm{DHEV} \mathrm{II})$

$$
\alpha=\frac{B_{\mathrm{DH}} \cdot \stackrel{\circ}{1-\frac{\mathrm{A}^{*}{ }_{\min }}{\left|z_{1} z_{2}\right| A_{\mathrm{DH}}}}}{}
$$

(The denominator in the DH-equation being $1+B_{\mathrm{DH}} \cdot \mathfrak{a} \cdot \sqrt{I}$. Eqn. (96) explains the proportionality between $\alpha$ and $R_{12}$ (corresponds to $a$ ) found in eqns. (93) and (94). However, $\stackrel{a}{a}$-values calculated from (96) and the above-mentioned eqns. are considerably larger than the DHEV II values, and the fit is not so precise in the dilute region as that of DHEV II (or the Guggenheim fits).

On Fig. 8, the ASPEV contact distances are shown as functions of temperature for $\mathrm{HCl}, \mathrm{HBr}, \mathrm{NaCl}, \mathrm{NaBr}, \mathrm{KCl}$ and $\mathrm{KBr}$ in aqueous solution. Data for $\ln \gamma_{ \pm}$as functions of temperature were found in Ref. 26, Appendix A, where formulae for $c_{\mathrm{s}} / m_{\mathrm{s}}$ at different temperatures were also found for the conversion to $\ln y_{ \pm} v s$ the square-root of the molar ionic strength $(\mathrm{KBr}$ was quite safely interpolated, however). Every point in Fig. 8 corresponds to a best fit to a whole table of $\ln y_{ \pm} v s . \sqrt{I}$. Some of the data $(\mathrm{HBr}$ and $\mathrm{KCl}$ ) deviate somewhat from other data at $25^{\circ} \mathrm{C}$, so the uncertainty on the radii is greater than apparent from the curves.

The ASPEV distances are somewhat greater than the ILEV II distances. For example,
$R_{12}($ ASPEV $)=5.08 \AA$ for $\mathrm{NaCl}$ at $25^{\circ} \mathrm{C}$ corresponding to $B($ ASPEV $)=0.33 \mathrm{dm}^{3} / \mathrm{mol}$. The value of $R_{12}$ (ILEV II) is $4.4 \AA$ corresponding to

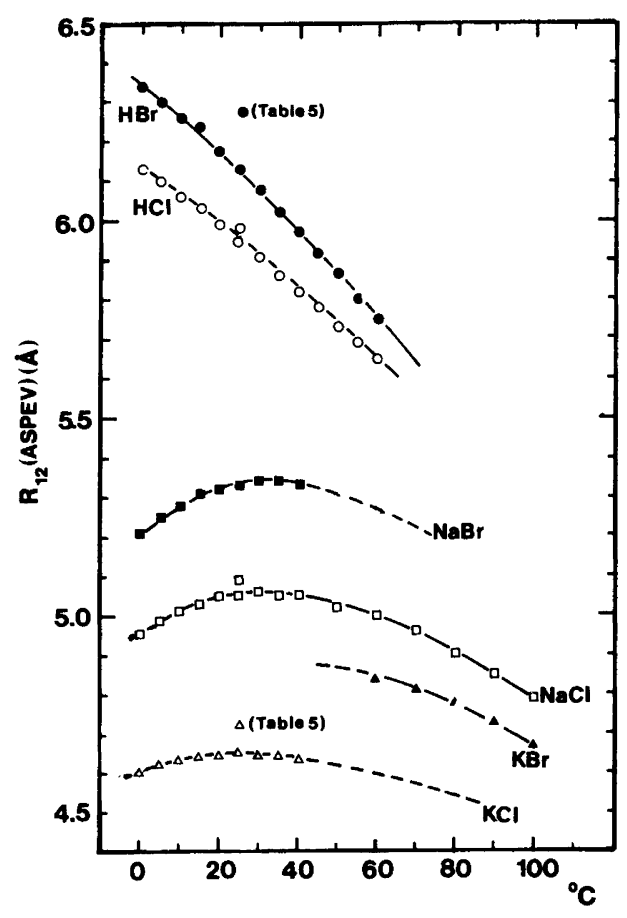

Fig. 8. ASPEV contact distances as a function of temperature for $\mathrm{HCl}, \mathrm{HBr}, \mathrm{NaCl}, \mathrm{NaBr}, \mathrm{KCl}$ and $\mathrm{KBr}$ in water. 
$B$ (ILEV) $=0.22 \mathrm{dm}^{3} / \mathrm{mol}$. The $B$-values may be compared without any regard to the validity of the Brønsted principle. Notice, that $B$ (ASPEV) falls in the middle of the range of $B$ (ILEV) calculated for $\mathrm{NaCl}$ from the macroscopic dielectric lowering (Fig. 7).

If we assume that $R_{12}(\mathrm{ASPEV})$ is approximately proportional to the real contact distances, Fig. 8 indicates the existence of a maximum in the contact distance around $30^{\circ} \mathrm{C}$, which is more pronounced for the sodium than for the potassium salts. The contact distances of $\mathrm{HCl}$ and $\mathrm{HBr}$ decrease monotonously with increasing temperature following the decrease in dielectric constant with increasing temperature (Fig. 9).

In an early, but quite comprehensive paper on the structure of water and ionic solutions, Bernal and Fowler ${ }^{48}$ have compiled arguments for the point of view, that water contains clusters of "quartz-like" structures (some "ice-tridymitelike" structures below $4{ }^{\circ} \mathrm{C}$ ) and a fraction of dipoles free to rotate. The decrease in dielectric constant with increasing temperature is described by a Langevin-equation with a Clausius-Mosotti equation for the "inner" electric field. The fraction of freely orientable water dipoles varies from 0.22 at $273 \mathrm{~K}$ to 0.28 at $350 \mathrm{~K}$, so in a certain sense the "quartz-like" structures "melt" and the increase in free dipoles compensates somewhat for the decrease in dielectric constant due to increasing thermal disorder (Langevin effect).

The proton may well be accomodated into the quartz-like clusters, and the relative great contact distances found with all models for the proton

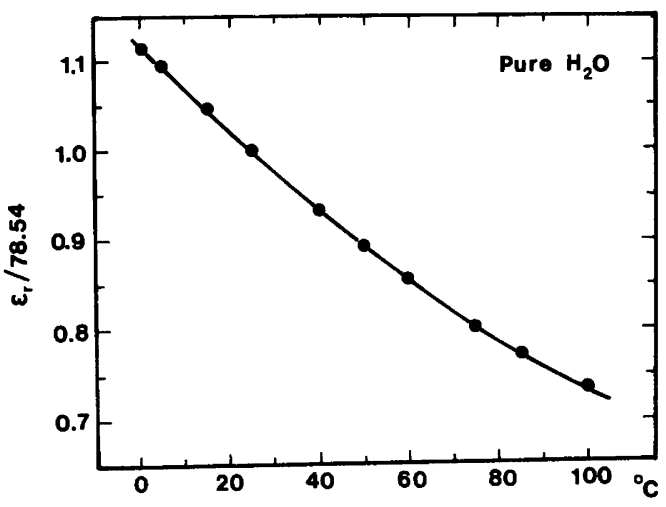

Fig. 9. Decrease in dielectric constant with increasing temperature for pure water according to formula (89). halogenides can be a reflection of the size of the quartz-like clusters. The decrease of $R_{12}$ (ASPEV) for $\mathrm{HCl}$ and $\mathrm{HBr}$ with increasing temperature should then reflect the "melting" of the "quartz-like" structures. On the other hand, the smaller alkali metal cations cannot be encompassed into the quartz-like structures. They have to make their own structure, binding water dipoles with electrostatic interactions. The electrostatic forces increase with decreasing dielectric constant of the solvent. Therefore, in the beginning $R_{12}$ (ASPEV) and the hydration layer thickness of the cation increases with increasing temperature. At higher temperatures, however, the hydration layer will also "melt" and $R_{12}$ (ASPEV) will decrease with temperature. We suggest this as a tentative explanation of the behaviour shown in Fig. 8. It should be evident from Fig. 8, that $\mathrm{Na}^{+}$has a thicker hydration layer than $\mathrm{K}^{+}$, so $R_{12}(\mathrm{ASPEV})$ is more sensitive to temperature for $\mathrm{Na}^{+}$than for $\mathrm{K}^{+}$.

Acknowledgement. We are grateful to Dr. J. B. Jensen for useful discussions.

\section{REFERENCES}

1. Milner, S. R. Philos. Mag. (6) 23 (1912) 551.

2. Milner, S. R. Philos. Mag. (6) 25 (1913) 742.

3. Milner, S. R. Philos. Mag. (6) 35 (1918) 214, 352.

4. Milner, S. R. Trans. Faraday Soc. 15 (1919) 148.

5. Debye, P. and Hückel, P. Phys. Zeitschr. 24 (1923) 185.

6. Mayer, J. E. J. Chem. Phys. 18 (1950) 1426.

7. Résibois, P. M. V. Electrolyte Theory, Harper and Row, New York, Evanston, London 1968, p. 47.

8. Schmitz, G. Phys. Lett. 21 (1966) 174.

9. Schmitz, G. Ann. Phys. 21 (1968) 31.

10. Falkenhagen, H. Theorie der Elektrolyte, Hirzel, Leipzig 1971.

11. Kirkwood, J. G. and Poirier, J. C. J. Phys. Chem. 58 (1954) 591.

12. McQuarrie, D. A. Statistical Mechanics, Harper and Row, New York, Evanston, San Francisco, London 1973.

13. Rasaiah, J. C. and Friedman, H. L. J. Chem. Phys. 48 (1968) 2742.

14. Rasaiah, J. C. and Friedman, H. L. J. Chem. Phys. 50 (1969) 3965.

15. Rasaiah, J. C. Chem. Phys. Lett. 7 (1970) 260. 
16. Rasaiah, J. C. J. Chem. Phys. 52 (1970) 704.

17. Card, D. N. and Valleau, J. P. J. Chem. Phys. 52 (1970) 6232.

18. Lebowitz, J. L. and Percus, J. K. Phys. Rev. 144 (1966) 251.

19. Waisman, E. and Lebowitz, J. L. J. Chem. Phys. 52 (1970) 4307; 56 (1972) 3086, 3093.

20. Guggenheim, E. A. and Turgeon, J. C. Trans. Faraday Soc. 51 (1955) 747.

21. Guggenheim, E. A. and Stokes, R. H. Trans. Faraday Soc. 54 (1958) 1646.

22. Brønsted, J. N. K. Dan. Vidensk. Selsk., Mat.-Fys. Medd. IV (1921) 4.

23. Brønsted, J. N. J. Am. Chem. Soc. 44 (1922) 877.

24. Sørensen, T. S. Acta Chem. Scand. A 32 (1978) 571.

25. Sørensen, T. S. Acta Chem. Scand. A 33 (1979) 583.

26. Harned, H. S. and Owen, B. B. The Physical Chemistry of Electrolyte Solutions, 3rd Ed., Van Nostrand-Reinhold, New York 1958.

27. Bjerrum, N. K. Dan. Vidensk. Selsk., Mat.Fys. Medd. VII, 9 (1926) 1.

28. Cohen, E. G. D. The Kinetic Theory of Moderately Dense Gases, In Hanley, H. J. M., Ed., Transport Phenomena in Fluids, Dekker, N.Y. 1969, Chapter 7.

29. Triolo, R., Grigera, J. R. and Blum. L. J. Phys. Chem. 80 (1976) 1858.

30. Blum, L. and Høye, J. S. J. Phys. Chem. 81 (1977) 1311.

31. Mayer, J. E. J. Chem. Phys. 10 (1942) 629.

32. Lewis, G. N. and Randall, M. J. Am. Chem. Soc. 43 (1921) 1112.

33. Newman, J. S. Electrochemical Systems, Prentice-Hall, Englewood Cliffs, N. J. 1973.

34. Weast, R. C. CRC Handbook of Chemistry and Physics, 58th Ed., CRC, West Palm Beach, Florida 1977-1978, F-213.

35. Ghosch, J. C. J. Chem. Soc. 113 (1918) 449.

36. Bjerrum, N. Z. Elektrochem. 24 (1918) 321.

37. Ghosch, J. C. Z. Phys. Chem. (Leipzig) 98 (1921) 211.

38. Prigogine, I. Contributions a la Theorie des Electrolytes Forts, Gauthiers-Villars, Paris 1939, Chapter 5.

39. Frank, H. S. and Thompson, P. T. J. Chem. Phys. 31 (1959) 1086.

40. Desnoyers, J. E. and Conway, B. E. J. Phys. Chem. 68 (1964) 2305.

41. Glueckauf, E. Proc. R. Soc. London, Ser. A 310 (1969) 449.

42. Bahe, L. W. J. Phys. Chem. 76 (1972) 1062.

43. Pytkowicz, R. M. and Johnson, K. S. Lattice Theories and a New Lattice Concept for Ionic Solutions, In Pytkowicz, R. M., Ed., Activity. Coefficients in Electrolyte Solutions, CRC, Boca Raton, Florida 1979, Vol. 1, Chapter 8.
44. Zhdanov, G. S. Crystal Physics, Oliver \& Boyd, Edinburg, London 1965.

45. Hückel, E. Phys. Zeitschr. 26 (1925) 93.

46. Robinson, R. A. and Stokes, R. H. Electrolyte Solutions, Butterworths, London 1968, Chapter 1.

47. Sørensen, T. S. and Jensen, J. B. Larebog $i$ Elektrokemi, Polyteknisk Forlag, Lyngby 1983.

48. Bernal, J. D. and Fowler, R. H. J. Chem. Phys. 1 (1933) 515.

49. Ruff, I. J. Chem. Soc. Faraday Trans. 2, 73 (1977) 1858.

50. Ruff, I. J. Chem. Soc. Faraday Trans. 2, 75 (1979) 1.

51. Ruff, I., Pálinkás, G. and Gombos, K. J. Chem. Soc. Faraday Trans. 2, 77 (1981) 1189.

52. Ruff. I. J. Chem. Soc. Faraday Trans. 2, 78 (1982) 617.

53. Booth, F. J. Chem. Phys. 19 (1951) 391, 1327, 1615.

54. Laidler, K. J. Can. J. Chem. 37 (1959) 138.

Received February 29, 1984. 